\title{
Data report: coring disturbances in IODP Expedition 340, a detailed list of intervals with fall-in and flow-in'
}

M. Jutzeler, ${ }^{2,3,4}$ P.J., Talling, ${ }^{2}$ J.D.L. White, ${ }^{4}$ and the Expedition 340 Scientists $^{5}$

\section{Chapter contents}

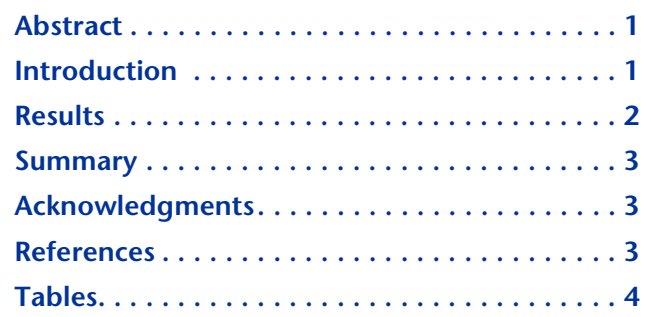

'Jutzeler, M., Talling, P.J., White, J.D.L., and the Expedition 340 Scientists, 2016. Data report: coring disturbances in IODP Expedition 340, a detailed list of intervals with fall-in and flow-in. In Le Friant, A., Ishizuka, O., Stroncik, N.A., and the Expedition 340 Scientists, Proceedings of the Integrated Ocean Drilling Program, 340: Tokyo (Integrated Ocean Drilling Program Management International, Inc.).

doi:10.2204/iodp.proc.340.206.2016

${ }^{2}$ National Oceanography Centre, Southampton, European Road, Waterfront Campus, SO14 3ZH

Southampton, United Kingdom. Correspondence author: jutzeler@gmail.com

${ }^{3}$ School of Physical Sciences-Earth Sciences, University of Tasmania, Private Bag 79, Hobart, TAS 7001, Australia.

${ }^{4}$ Geology Department, PO Box 56, University of Otago, Dunedin 9001, New Zealand.

${ }^{5}$ Expedition 340 Scientists' addresses.

\section{Abstract}

We report an exhaustive list of coring disturbances from the entire collection of piston cores collected during Integrated Ocean Drilling Program (IODP) Expedition 340. Expedition 340 cores contain numerous coring disturbances resulting from the particularly thick and coarse granular material cored. Overall, 23\%-32\% of the recovered core length is disturbed. Here, coring disturbances are grouped into fall-in and flow-in classes, and uncertain intervals are distinguished from those with clear evidence of coring disturbances. Fall-in corresponds to fallen material atop the core, chiefly from hole collapse. Flow-in includes basal flow-in, which occurs at the bottom of a core, and midcore flow-in, which corresponds to injection of sand into the in situ core stratigraphy. Basal flow-in is likely to occur in conditions of partial stroke, whereas midcore flow-in seems to occur where the core material was stretched during recovery.

\section{Introduction}

In specific types of sediments, Integrated Ocean Drilling Program (IODP) advanced piston corer (APC) technology can produce substantial coring disturbances. These disturbances are mostly present where thick intervals of loose, granular, unconsolidated sediment occur. Volcaniclastic deposits are the sediment type most prone to being affected because they are commonly very thick and well-sorted deposits with particles of low density and low cohesion. Coring disturbances can affect large parts of the core, up to total disruption of the original stratigraphy. More details of about specific types of coring disturbances are found in Jutzeler et al. (2014).

We consider here only two types of coring disturbances, the ones most likely to destroy the original stratigraphy: fall-in and flowin. Fall-in refers to material that has fallen into the hole or did not get washed during drilling and hole cleaning and was then collected at the top of the next core. These disturbances are commonly easy to identify because of their position at the uppermost part of the core and their common coarse-grained textures. In contrast, flow-in disturbances can affect a few centimeters to the entire length of the core, and the resulting facies may be extremely similar to some natural normally graded facies. Basal flow-in is restricted to the lower part of the core, whereas midcore 
flow-in can occur at any position. Cores disturbed by flow-in may have been subsequently disturbed when put horizontally on deck (Jutzeler et al., 2014). We do not consider disturbance by core extension (Jutzeler et al., 2014) because it generally does not mix several intervals together.

Because of the intrinsic difficulties in determining where core disturbances are most likely to occur, we analyzed every high-resolution image of core sections from Expedition 340: 277 cores and 1818 core sections. This list of disturbed intervals does not include reconstruction of the stratigraphy between holes, which would in many cases require an extensive study including componentry, dating, and chemical analyses. The present exhaustive list will allow researchers to study and interpret Expedition 340 cores with much more confidence. Importantly, it will help researchers involved in future studies to avoid parts of the cores that are not representative of the original stratigraphy.

\section{Results}

Of the 1818 core sections recovered during Expedition 340 (Expedition 340 Scientists, 2013), 36\% contain fall-in or flow-in coring disturbances and $23 \%$ $32 \%$ of the total recovered core length is made of disturbed sediment (Tables T1, T2). The present data set shows that a large number of coring disturbances occur because of partial stroke (Jutzeler et al., 2014). Without considering failed holes (U1393A and U1396B), the cores from Montserrat (minimum $11.4 \%$ and maximum $13.2 \%$ of core length) are much less disturbed than those from Martinique (minimum $25.3 \%$ and maximum $30.6 \%$ of core length). This high percentage is probably due to the high abundance of volcaniclastic intervals offshore Martinique. Moreover, the relatively high shear stress of the hemipelagic mud at Martinique sites (Expedition 340 Scientists, 2013) likely increased friction of the host formation on the core barrel. High friction rates on the core barrel (1) reduce the overall velocity of the APC during penetration, favoring partial strokes, and (2) increase the tension on the core barrel when pulled out from the host formation, favoring flow-in.

\section{Fall-in}

Fall-in is commonly identified with coarse, ungraded to normally graded intervals at the uppermost part of a core section (Jutzeler et al., 2014). Where cores are entirely disturbed (e.g., Core 340-U1400B-5H), it is more difficult to assess whether and where there is a contact between fall-in and flow-in disturbances, but given such complete disturbance the distinction becomes irrelevant.

\section{Flow-in}

Basal flow-in is commonly easily identified from the presence of loose, soupy, commonly normally graded sand at the base of the core (Jutzeler et al., 2014). In some places, preserved halos of alteration with gradients of intensity (e.g., glaucony or dark alteration) coronae around clasts allow identification of nondisturbed domains.

Midcore flow-in, which can occur with or without basal flow-in, is identified where floating disaggregated pieces of hemipelagic mud occur in a loose volcaniclastic sand (Jutzeler et al., 2014) but should not be misidentified with primary textures of floating mud intraclasts in volcaniclastic sand. In other places, midcore flow-in is identified where sand of the same apparent componentry was injected into cracks in intervals of more cohesive material (commonly hemipelagic mud) or as the occurrence of a thin rim along the core liner (Jutzeler et al., 2014). Cracks in the mud are likely to be formed during initial recovery (pull) of the core from the formation when the core may be stretched along its entire length. In some cores, it becomes very difficult to distinguish midcore flow-in from local core extension (affecting a single interval) or from genuine mixing by creep or slump (e.g., Sections 340U1398B-18H-4, 20-145 cm, and 340-U1399A-23H-3, $62 \mathrm{~cm}$, to $23 \mathrm{H}-5,140 \mathrm{~cm}$ ).

\section{Analyses on disturbed cores}

Depending on the intensity and type of coring disturbances, some parts of the disturbed intervals may still be available for specific analyses. We give three examples:

1. The stratigraphy within an interval of flowed-in volcaniclastic sand may be destroyed, but bulk componentry and dating are still possible if the sample is considered as matching the entire length of the disturbance.

2. Similarly, if fall-in clasts are dated to the rough age of the preceding core, they may be available for analyses.

3. A piece of hemipelagic mud floating within volcaniclastic sand may still be used for oxygen isotope dating; however, its exact position in the stratigraphy of the core includes a large error, as loose sand may have been injected above it. 


\section{Summary}

All piston cores from Expedition 340 were analyzed for fall-in and flow-in coring disturbances. Volcaniclastic material favors formation of coring disturbances in APC coring operations, and a large number of Expedition 340 cores are affected (23\%-32\% of total recovered core length). The analysis presented here allows careful sample selection within the pristine intervals to avoid unrepresentative samples. Depending on the intensity of the coring disturbance, some types of analyses can still be carried out on part of the interval; however, we recommend extreme caution.

\section{Acknowledgments}

Core images were provided by the Integrated Ocean Drilling Program. Funding was provided by the Swiss National Science Foundation (grants PBSKP2_138556 and PBSKP2_145907) and research grants from the University of Otago, ANZIC, and IODP-UK. A review by C. Busby is acknowledged.

\section{References}

Expedition 340 Scientists, 2013. Expedition 340 summary. In Le Friant, A., Ishizuka, O., Stroncik, N.A., and the Expedition 340 Scientists, Proceedings of the Integrated Ocean Drilling Program, 340: Tokyo (Integrated Ocean Drilling Program Management International, Inc.). http://dx.doi.org/10.2204/iodp.proc.340.101.2013

Jutzeler, M., White, J.D.L., Talling, P.J., McCanta, M., Morgan, S., Le Friant, A., and Ishizuka, O., 2014. Coring disturbances in IODP piston cores with implications for offshore record of volcanic events and the Missoula megafloods. Geochemistry, Geophysics, Geosystems, 15(9):3572-3590.

http://dx.doi.org/10.1002/2014GC005447

Initial receipt: 30 July 2015

Acceptance: 17 November 2015

Publication: 12 February 2016

MS 340-206 
Table T1. Detailed identification of coring disturbances in Expedition 340 cores. (Continued on next ten pages.)

\begin{tabular}{|c|c|c|c|c|c|c|c|c|c|c|c|c|c|c|}
\hline \multirow{2}{*}{$\begin{array}{l}\text { Core, } \\
\text { section }\end{array}$} & \multirow{2}{*}{$\begin{array}{l}\text { Recovered } \\
\text { length } \\
(\mathrm{m})\end{array}$} & \multicolumn{2}{|c|}{ Depth CSF-A (m) } & \multicolumn{2}{|c|}{ Depth CSF-B (m) } & \multirow{2}{*}{$\begin{array}{l}\text { Partial } \\
\text { stroke }\end{array}$} & \multicolumn{2}{|c|}{$\begin{array}{c}\text { Fall-in }{ }^{*} \text { or flow-in } \\
\text { depth in section }(\mathrm{m}) \\
\end{array}$} & \multicolumn{2}{|c|}{$\begin{array}{l}\text { Possible fall-in* or } \\
\text { flow-in }^{\dagger} \text { depth } \\
\text { in section }(\mathrm{m})\end{array}$} & \multicolumn{2}{|c|}{$\begin{array}{l}\text { Fall-in* or flow-in }{ }^{\dagger} \\
\text { depth in hole } \\
\text { CSF-A }(m)\end{array}$} & \multicolumn{2}{|c|}{$\begin{array}{l}\text { Possible fall-in* or } \\
\text { flow-in }{ }^{\dagger} \text { depth } \\
\text { in hole CSF-A }(\mathrm{m})\end{array}$} \\
\hline & & Top & Bottom & Top & Bottom & & Top & Bottom & Top & Bottom & Top & Bottom & Top & Bottom \\
\hline $340-U 139=$ & & & & & & & & & & & & & & \\
\hline $1 \mathrm{H}-1$ & 1.5 & 0 & 1.5 & 0 & 1.48 & No & & & $0^{*}$ & $1.5^{*}$ & & & $0^{*}$ & $1.5^{*}$ \\
\hline $1 \mathrm{H}-2$ & 1.5 & 1.5 & 3 & 1.48 & 2.96 & No & & & $0^{*}$ & $1.5^{\star}$ & & & $1.5^{*}$ & $3^{*}$ \\
\hline $1 \mathrm{H}-3$ & 0.53 & 3 & 3.53 & 2.96 & 3.48 & No & $0^{*}$ & $0.53^{*}$ & & & $3^{*}$ & $3.53^{*}$ & & \\
\hline $1 \mathrm{H}-4$ & 0.71 & 3.53 & 4.24 & 3.48 & 4.18 & No & $0^{*}$ & $0.71^{*}$ & & & $3.53^{*}$ & $4.24^{\star}$ & & \\
\hline $1 \mathrm{H}-\mathrm{CC}$ & 0.12 & 4.24 & 4.36 & 4.18 & 4.3 & No & $0^{*}$ & $0.12^{*}$ & & & $4.24^{*}$ & $4.36^{*}$ & & \\
\hline $2 \mathrm{H}-\mathrm{CC}$ & 0.01 & 4.3 & 4.31 & 4.3 & 4.31 & Yes & $0^{\dagger}$ & $0.01^{\dagger}$ & & & $4.3^{\dagger}$ & $4.31^{\dagger}$ & & \\
\hline $340-U 139$ & & & & & & & & & & & & & & \\
\hline $2 \mathrm{H}-7$ & 0.87 & 13.57 & 14.44 & 13.3 & 14.14 & No & $0^{*}$ & $0.87^{*}$ & & & $13.57^{*}$ & $14.44^{*}$ & & \\
\hline $2 \mathrm{H}-\mathrm{CC}$ & 0.16 & 14.44 & 14.6 & 14.14 & 14.3 & No & $0^{*}$ & $0.16^{*}$ & & & $14.44^{*}$ & $14.6^{*}$ & & \\
\hline $3 \mathrm{H}-1$ & 1.5 & 14.3 & 15.8 & 14.3 & 15.8 & No & $0.55^{*}$ & $1.5^{*}$ & & & $14.85^{*}$ & $15.8^{*}$ & & \\
\hline $3 \mathrm{H}-2$ & 1.5 & 15.8 & 17.3 & 15.8 & 17.3 & No & $0^{*}$ & $1.5^{*}$ & & & $15.8^{*}$ & $17.3^{*}$ & & \\
\hline $3 \mathrm{H}-3$ & 1.5 & 17.3 & 18.8 & 17.3 & 18.8 & No & $0^{*}$ & $1.5^{*}$ & & & $17.3^{*}$ & $18.8^{*}$ & & \\
\hline $3 \mathrm{H}-4$ & 1.5 & 18.8 & 20.3 & 18.8 & 20.3 & No & $0^{*}$ & $1.5^{*}$ & & & $18.8^{*}$ & $20.3^{*}$ & & \\
\hline $3 \mathrm{H}-5$ & 1.5 & 20.3 & 21.8 & 20.3 & 21.8 & No & $0^{*}$ & $1.5^{*}$ & & & $20.3^{*}$ & $21.8^{*}$ & & \\
\hline $3 \mathrm{H}-6$ & 1.11 & 21.8 & 22.91 & 21.8 & 22.91 & No & $0^{*}$ & $1.11^{*}$ & & & $21.8^{*}$ & $22.91^{*}$ & & \\
\hline $3 \mathrm{H}-7$ & 0.64 & 22.91 & 23.55 & 22.91 & 23.55 & No & $0^{*}$ & $0.64^{*}$ & & & $22.91^{*}$ & $23.55^{*}$ & & \\
\hline $3 \mathrm{H}-\mathrm{CC}$ & 0.17 & 23.55 & 23.72 & 23.55 & 23.72 & No & $0^{*}$ & $0.17^{*}$ & & & $23.55^{*}$ & $23.72^{*}$ & & \\
\hline $4 \mathrm{H}-\mathrm{CC}$ & 0.1 & 23.8 & 23.9 & 23.8 & 23.9 & Yes & $0^{*}$ & $0.1^{*}$ & & & $23.8^{\star}$ & $23.9^{*}$ & & \\
\hline $340-U 139$ & & & & & & & & & & & & & & \\
\hline $8 \mathrm{H}-1$ & 1.5 & 59.8 & 61.3 & 59.8 & 61.29 & Yes & $0^{\dagger}$ & $0.56^{\dagger}$ & & & $59.8^{\dagger}$ & $60.36^{\dagger}$ & & \\
\hline $8 \mathrm{H}-2$ & 1.17 & 61.3 & 62.47 & 61.29 & 62.46 & Yes & $0.78^{*}$ & $1.17^{*}$ & & & $62.08^{*}$ & $62.47^{*}$ & & \\
\hline $8 \mathrm{H}-3$ & 1.22 & 62.47 & 63.69 & 62.46 & 63.67 & Yes & $0^{*}$ & $1.22^{\star}$ & & & $62.47^{*}$ & $63.69^{*}$ & & \\
\hline $8 \mathrm{H}-4$ & 1.21 & 63.69 & 64.9 & 63.67 & 64.88 & Yes & $0^{*}$ & $1.21^{*}$ & & & $63.69^{*}$ & $64.9^{*}$ & & \\
\hline $8 \mathrm{H}-5$ & 1.27 & 64.9 & 66.17 & 64.88 & 66.14 & Yes & $0^{*}$ & $1.27^{*}$ & & & $64.9^{*}$ & $66.17^{*}$ & & \\
\hline $8 \mathrm{H}-\mathrm{CC}$ & 0.16 & 66.17 & 66.33 & 66.14 & 66.3 & Yes & $0^{*}$ & $0.16^{\star}$ & & & $66.17^{*}$ & $66.33^{*}$ & & \\
\hline $9 \mathrm{H}-1$ & 1.5 & 66.3 & 67.8 & 66.3 & 67.8 & Yes & $0^{\dagger}$ & $0.18^{\dagger}$ & & & $66.3^{\dagger}$ & $66.48^{\dagger}$ & & \\
\hline $9 \mathrm{H}-4$ & 1.2 & 69.51 & 70.71 & 69.51 & 70.71 & Yes & $0^{*}$ & $1.2^{*}$ & & & $69.51^{*}$ & $70.71^{*}$ & & \\
\hline $9 \mathrm{H}-5$ & 0.74 & 70.71 & 71.45 & 70.71 & 71.45 & Yes & $0^{*}$ & $0.74^{*}$ & & & $70.71^{*}$ & $71.45^{*}$ & & \\
\hline 9H-CC & 0.21 & 71.45 & 71.66 & 71.45 & 71.66 & Yes & $0^{*}$ & $0.21^{*}$ & & & $71.45^{*}$ & $71.66^{*}$ & & \\
\hline $10 \mathrm{H}-1$ & 1.5 & 72.5 & 74 & 72.5 & 73.99 & Yes & $0^{\dagger}$ & $0.2^{\dagger}$ & & & $72.5^{\dagger}$ & $72.7^{\dagger}$ & & \\
\hline $10 \mathrm{H}-3$ & 1.5 & 75.5 & 77 & 75.48 & 76.97 & Yes & $1.15^{*}$ & $1.5^{\star}$ & & & $76.65^{*}$ & $77^{*}$ & & \\
\hline $10 \mathrm{H}-4$ & 1.5 & 77 & 78.5 & 76.97 & 78.46 & Yes & $0^{*}$ & $1.5^{*}$ & & & $77^{*}$ & $78.5^{*}$ & & \\
\hline $10 \mathrm{H}-5$ & 1.5 & 78.5 & 80 & 78.46 & 79.95 & Yes & $0^{*}$ & $1.5^{*}$ & & & $78.5^{*}$ & $80^{*}$ & & \\
\hline $10 \mathrm{H}-6$ & 1.2 & 80 & 81.2 & 79.95 & 81.14 & Yes & $0^{*}$ & $1.2^{*}$ & & & $80^{*}$ & $81.2^{*}$ & & \\
\hline $10 \mathrm{H}-7$ & 0.7 & 81.2 & 81.9 & 81.14 & 81.84 & Yes & $0^{*}$ & $0.7^{*}$ & & & $81.2^{*}$ & $81.9^{*}$ & & \\
\hline $10 \mathrm{H}-\mathrm{CC}$ & 0.16 & 81.9 & 82.06 & 81.84 & 82 & Yes & $0^{*}$ & $0.16^{*}$ & & & $81.9^{*}$ & $82.06^{*}$ & & \\
\hline $12 \mathrm{H}-1$ & 1.5 & 91.5 & 93 & 91.5 & 92.94 & Yes & $0^{\dagger}$ & $0.28^{\dagger}$ & & & $91.5^{\dagger}$ & $91.78^{\dagger}$ & & \\
\hline $12 \mathrm{H}-4$ & 1.5 & 96 & 97.5 & 95.83 & 97.27 & Yes & $0.67^{*}$ & $1.5^{\star}$ & & & $96.67^{*}$ & $97.5^{\star}$ & & \\
\hline $12 \mathrm{H}-5$ & 1.5 & 97.5 & 99 & 97.27 & 98.71 & Yes & $0^{*}$ & $1.5^{*}$ & & & $97.5^{*}$ & $99^{*}$ & & \\
\hline $12 \mathrm{H}-6$ & 1.5 & 99 & 100.5 & 98.71 & 100.15 & Yes & $0^{*}$ & $1.5^{*}$ & & & $99^{*}$ & $100.5^{*}$ & & \\
\hline $12 \mathrm{H}-7$ & 0.68 & 100.5 & 101.18 & 100.15 & 100.81 & Yes & $0^{*}$ & $0.68^{*}$ & & & $100.5^{*}$ & $101.18^{*}$ & & \\
\hline $12 \mathrm{H}-\mathrm{CC}$ & 0.2 & 101.18 & 101.38 & 100.81 & 101 & Yes & $0^{*}$ & $0.2^{*}$ & & & $101.18^{*}$ & $101.38^{*}$ & & \\
\hline $13 \mathrm{H}-1$ & 1.5 & 101 & 102.5 & 101 & 102.49 & Yes & $0^{\dagger}$ & $0.15^{\dagger}$ & & & $101^{\dagger}$ & $101.15^{\dagger}$ & & \\
\hline $13 \mathrm{H}-1$ & 1.5 & 101 & 102.5 & 101 & 102.49 & Yes & $0.15^{*}$ & $1.5^{\star}$ & & & $101.15^{*}$ & $102.5^{*}$ & & \\
\hline $13 \mathrm{H}-2$ & 1.5 & 102.5 & 104 & 102.49 & 103.98 & Yes & $0^{*}$ & $1.5^{\star}$ & & & $102.5^{*}$ & $104^{*}$ & & \\
\hline $13 \mathrm{H}-3$ & 1.5 & 104 & 105.5 & 103.98 & 105.48 & Yes & $0^{*}$ & $1.5^{*}$ & & & $104^{*}$ & $105.5^{*}$ & & \\
\hline $13 \mathrm{H}-4$ & 1.5 & 105.5 & 107 & 105.48 & 106.97 & Yes & $0^{*}$ & $1.5^{*}$ & & & $105.5^{*}$ & $107^{*}$ & & \\
\hline $13 \mathrm{H}-5$ & 1.5 & 107 & 108.5 & 106.97 & 108.46 & Yes & $0^{*}$ & $1.5^{\star}$ & & & $107^{*}$ & $108.5^{*}$ & & \\
\hline $13 \mathrm{H}-6$ & 1.38 & 108.5 & 109.88 & 108.46 & 109.83 & Yes & $0^{*}$ & $1.38^{\star}$ & & & $108.5^{*}$ & $109.88^{*}$ & & \\
\hline $13 \mathrm{H}-\mathrm{CC}$ & 0.17 & 109.88 & 110.05 & 109.83 & 110 & Yes & $0^{*}$ & $0.17^{*}$ & & & $109.88^{*}$ & $110.05^{*}$ & & \\
\hline $14 \mathrm{H}-1$ & 1.5 & 110 & 111.5 & 110 & 111.5 & No & $0^{\dagger}$ & $0.14^{\dagger}$ & & & $110^{\dagger}$ & $110.14^{\dagger}$ & & \\
\hline $19 \mathrm{H}-1$ & 1.25 & 153.7 & 154.95 & 153.7 & 154.95 & Yes & $1.02^{*}$ & $1.25^{\star}$ & & & $154.72^{\star}$ & $154.95^{*}$ & & \\
\hline $19 \mathrm{H}-2$ & 1.5 & 154.95 & 156.45 & 154.95 & 156.45 & Yes & $0^{*}$ & $1.5^{*}$ & & & $154.95^{*}$ & $156.45^{*}$ & & \\
\hline $19 \mathrm{H}-3$ & 1.5 & 156.45 & 157.95 & 156.45 & 157.95 & Yes & $0^{*}$ & $1.5^{*}$ & & & $156.45^{*}$ & $157.95^{*}$ & & \\
\hline $19 \mathrm{H}-4$ & 1.5 & 157.95 & 159.45 & 157.95 & 159.45 & Yes & $0^{*}$ & $1.5^{*}$ & & & $157.95^{*}$ & $159.45^{*}$ & & \\
\hline $19 \mathrm{H}-5$ & 1.5 & 159.45 & 160.95 & 159.45 & 160.95 & Yes & $0^{*}$ & $1.5^{\star}$ & & & $159.45^{*}$ & $160.95^{*}$ & & \\
\hline $19 \mathrm{H}-6$ & 1.5 & 160.95 & 162.45 & 160.95 & 162.45 & Yes & $0^{*}$ & $1.5^{\star}$ & & & $160.95^{*}$ & $162.45^{*}$ & & \\
\hline $19 \mathrm{H}-7$ & 0.63 & 162.45 & 163.08 & 162.45 & 163.08 & Yes & $0^{*}$ & $0.63^{*}$ & & & $162.45^{*}$ & $163.08^{*}$ & & \\
\hline $19 \mathrm{H}-\mathrm{CC}$ & 0.1 & 163.08 & 163.18 & 163.08 & 163.18 & Yes & $0^{*}$ & $0.1^{*}$ & & & $163.08^{*}$ & $163.18^{*}$ & & \\
\hline $20 \mathrm{H}-1$ & 1.5 & 163.2 & 164.7 & 163.2 & 164.69 & Yes & & & $0^{\dagger}$ & $0.07^{\dagger}$ & & & $163.2^{\dagger}$ & $163.27^{\dagger}$ \\
\hline $21 \mathrm{H}-1$ & 1.5 & 172.7 & 174.2 & 172.7 & 174.2 & Yes & & & $0^{\dagger}$ & $0.05^{\dagger}$ & & & $172.7^{\dagger}$ & $172.75^{\dagger}$ \\
\hline $21 \mathrm{H}-4$ & 1.15 & 177.03 & 178.18 & 177.03 & 178.18 & Yes & $0^{*}$ & $1.15^{\star}$ & & & $177.03^{*}$ & $178.18^{*}$ & & \\
\hline $21 \mathrm{H}-5$ & 1.12 & 178.18 & 179.3 & 178.18 & 179.3 & Yes & $0^{*}$ & $1.12^{*}$ & & & $178.18^{*}$ & $179.3^{*}$ & & \\
\hline
\end{tabular}


Table T1 (continued). (Continued on next page.)

\begin{tabular}{|c|c|c|c|c|c|c|c|c|c|c|c|c|c|c|}
\hline \multirow{2}{*}{$\begin{array}{l}\text { Core, } \\
\text { section }\end{array}$} & \multirow{2}{*}{$\begin{array}{l}\text { Recovered } \\
\text { length } \\
(\mathrm{m})\end{array}$} & \multicolumn{2}{|c|}{ Depth CSF-A (m) } & \multicolumn{2}{|c|}{ Depth CSF-B (m) } & \multirow{2}{*}{$\begin{array}{l}\text { Partial } \\
\text { stroke }\end{array}$} & \multicolumn{2}{|c|}{$\begin{array}{c}\text { Fall-in* or flow-in } \\
\text { depth in section }(\mathrm{m}) \\
\end{array}$} & \multicolumn{2}{|c|}{$\begin{array}{l}\text { Possible fall-in* or } \\
\text { flow-in }{ }^{\dagger} \text { depth } \\
\text { in section }(\mathrm{m}) \\
\end{array}$} & \multicolumn{2}{|c|}{$\begin{array}{l}\text { Fall-in* or flow-in }{ }^{\dagger} \\
\text { depth in hole } \\
\text { CSF-A (m) } \\
\end{array}$} & $\begin{array}{r}\text { Possible f } \\
\text { flow-in' } \\
\text { in hole C } \\
\end{array}$ & $\begin{array}{l}\text { all-in* or } \\
\text { depth } \\
\text { SF-A }(m)\end{array}$ \\
\hline & & Top & Bottom & Top & Bottom & & Top & Bottom & Top & Bottom & Top & Bottom & Top & Bottom \\
\hline $21 \mathrm{H}-6$ & 1.34 & 179.3 & 180.64 & 179.3 & 180.64 & Yes & $0^{*}$ & $1.34^{*}$ & & & $179.3^{*}$ & $180.64^{*}$ & & \\
\hline $21 \mathrm{H}-7$ & 0.66 & 180.64 & 181.3 & 180.64 & 181.3 & Yes & $0^{*}$ & $0.66^{*}$ & & & $180.64^{*}$ & $181.3^{*}$ & & \\
\hline $21 \mathrm{H}-\mathrm{CC}$ & 0.1 & 181.3 & 181.4 & 181.3 & 181.4 & Yes & $0^{*}$ & $0.1^{*}$ & & & $181.3^{*}$ & $181.4^{*}$ & & \\
\hline $340-U 1395$ & & & & & & & & & & & & & & \\
\hline $3 \mathrm{H}-4$ & 1.5 & 16.83 & 18.33 & 16.82 & 18.32 & Yes & & & $0.3^{*}$ & $1.5^{*}$ & & & $17.13^{*}$ & $18.33^{*}$ \\
\hline $3 \mathrm{H}-5$ & 1.42 & 18.33 & 19.75 & 18.32 & 19.74 & Yes & & & $0^{*}$ & $1.42^{*}$ & & & $18.33^{*}$ & $19.75^{*}$ \\
\hline $3 \mathrm{H}-\mathrm{CC}$ & 0.16 & 19.75 & 19.91 & 19.74 & 19.9 & Yes & & & $0^{*}$ & $0.16^{*}$ & & & $19.75^{*}$ & 19.91* \\
\hline $6 \mathrm{H}-1$ & 1.51 & 38.9 & 40.41 & 38.9 & 40.32 & No & & & $0^{\dagger}$ & $0.09^{\dagger}$ & & & $38.9^{\dagger}$ & $38.99^{\dagger}$ \\
\hline $7 \mathrm{H}-3$ & 1.5 & 51.4 & 52.9 & 51.39 & 52.88 & Yes & & & $0.63^{*}$ & $1.5^{*}$ & & & $52.03^{*}$ & $52.9^{*}$ \\
\hline $7 \mathrm{H}-4$ & 1.5 & 52.9 & 54.4 & 52.88 & 54.37 & Yes & & & $0^{*}$ & $1.5^{*}$ & & & $52.9^{*}$ & $54.4^{*}$ \\
\hline $7 \mathrm{H}-5$ & 1.08 & 54.4 & 55.48 & 54.37 & 55.45 & Yes & & & $0^{*}$ & $1.08^{*}$ & & & $54.4^{*}$ & $55.48^{*}$ \\
\hline $7 \mathrm{H}-6$ & 1.5 & 55.48 & 56.98 & 55.45 & 56.94 & Yes & & & $0^{*}$ & $1.5^{*}$ & & & $55.48^{*}$ & $56.98^{*}$ \\
\hline 7H-CC & 0.16 & 56.98 & 57.14 & 56.94 & 57.1 & Yes & & & $0^{*}$ & $0.16^{*}$ & & & $56.98^{*}$ & $57.14^{*}$ \\
\hline $14 \mathrm{H}-3$ & 1.31 & 111.9 & 113.21 & 111.89 & 113.2 & Yes & $0.52^{*}$ & $1.31^{*}$ & & & $112.42^{*}$ & $113.21^{*}$ & & \\
\hline $14 \mathrm{H}-4$ & 0.74 & 113.21 & 113.95 & 113.2 & 113.94 & Yes & $0^{*}$ & $0.74^{*}$ & & & $113.21^{*}$ & $113.95^{*}$ & & \\
\hline $14 \mathrm{H}-5$ & 1.5 & 113.95 & 115.45 & 113.94 & 115.43 & Yes & $0^{*}$ & $1.5^{*}$ & & & $113.95^{*}$ & $115.45^{*}$ & & \\
\hline $14 \mathrm{H}-6$ & 1.38 & 115.45 & 116.83 & 115.43 & 116.81 & Yes & $0^{*}$ & $1.38^{*}$ & & & $115.45^{*}$ & $116.83^{*}$ & & \\
\hline $14 \mathrm{H}-\mathrm{CC}$ & 0.09 & 116.83 & 116.92 & 116.81 & 116.9 & Yes & $0^{*}$ & $0.09^{*}$ & & & $116.83^{*}$ & $116.92^{*}$ & & \\
\hline $15 \mathrm{H}-1$ & 1.5 & 116.9 & 118.4 & 116.9 & 118.37 & Yes & $0^{\dagger}$ & $0.3^{\dagger}$ & & & $116.9^{\dagger}$ & $117.2^{\dagger}$ & & \\
\hline $15 \mathrm{H}-1$ & 1.5 & 116.9 & 118.4 & 116.9 & 118.37 & Yes & $0.35^{*}$ & $1.5^{*}$ & & & $117.25^{*}$ & $118.4^{*}$ & & \\
\hline $15 \mathrm{H}-2$ & 1.5 & 118.4 & 119.9 & 118.37 & 119.85 & Yes & $0^{*}$ & $1.5^{*}$ & & & $118.4^{*}$ & 119.9* & & \\
\hline $15 \mathrm{H}-3$ & 0.79 & 119.9 & 120.69 & 119.85 & 120.62 & Yes & $0^{*}$ & $0.79^{*}$ & & & $119.9^{*}$ & $120.69^{*}$ & & \\
\hline $15 \mathrm{H}-\mathrm{CC}$ & 0.18 & 120.69 & 120.87 & 120.62 & 120.8 & Yes & $0^{*}$ & $0.18^{*}$ & & & $120.69 *$ & $120.87^{*}$ & & \\
\hline $340-U 1395$ & & & & & & & & & & & & & & \\
\hline $2 \mathrm{H}-5$ & 1.5 & 11.9 & 13.4 & 11.82 & 13.3 & No & $0^{*}$ & $1.5^{*}$ & & & $11.9^{*}$ & $13.4^{*}$ & & \\
\hline $2 \mathrm{H}-6$ & 1.3 & 13.4 & 14.7 & 13.3 & 14.58 & No & $0^{*}$ & $1.3^{*}$ & & & $13.4^{*}$ & $14.7^{*}$ & & \\
\hline $2 \mathrm{H}-7$ & 0.65 & 14.7 & 15.35 & 14.58 & 15.22 & No & $0^{*}$ & $0.65^{*}$ & & & $14.7^{*}$ & $15.35^{*}$ & & \\
\hline $2 \mathrm{H}-\mathrm{CC}$ & 0.18 & 15.35 & 15.53 & 15.22 & 15.4 & No & $0^{*}$ & $0.18^{*}$ & & & $15.35^{*}$ & $15.53^{*}$ & & \\
\hline $3 \mathrm{H}-1$ & 1.5 & 15.4 & 16.9 & 15.4 & 16.84 & No & & & $0^{\dagger}$ & $1.5^{\dagger}$ & & & $15.4^{\dagger}$ & $16.9^{\dagger}$ \\
\hline $3 \mathrm{H}-2$ & 1.5 & 16.9 & 18.4 & 16.84 & 18.29 & No & & & $0^{\dagger}$ & $1.46^{\dagger}$ & & & $16.9^{\dagger}$ & $18.36^{\dagger}$ \\
\hline $8 \mathrm{H}-6$ & 1.3 & 69.08 & 70.38 & 69.06 & 70.36 & Yes & $0^{*}$ & $1.3^{*}$ & & & $69.08^{*}$ & $70.38^{*}$ & & \\
\hline $8 \mathrm{H}-7$ & 0.72 & 70.38 & 71.1 & 70.36 & 71.08 & Yes & $0^{*}$ & $0.72^{*}$ & & & $70.38^{*}$ & $71.1^{*}$ & & \\
\hline $8 \mathrm{H}-\mathrm{CC}$ & 0.12 & 71.1 & 71.22 & 71.08 & 71.2 & Yes & $0^{*}$ & $0.12^{*}$ & & & $71.1^{*}$ & $71.22^{*}$ & & \\
\hline $9 \mathrm{H}-2$ & 1.34 & 72.7 & 74.04 & 72.69 & 74.02 & Yes & $1^{*}$ & $1.34^{*}$ & & & $73.7^{*}$ & $74.04^{*}$ & & \\
\hline $9 \mathrm{H}-3$ & 1.36 & 74.04 & 75.4 & 74.02 & 75.37 & Yes & $0^{*}$ & $1.36^{*}$ & & & $74.04^{*}$ & $75.4^{\star}$ & & \\
\hline $9 \mathrm{H}-4$ & 1.15 & 75.4 & 76.55 & 75.37 & 76.51 & Yes & $0^{*}$ & $1.15^{*}$ & & & $75.4^{\star}$ & $76.55^{*}$ & & \\
\hline $9 \mathrm{H}-5$ & 0.72 & 76.55 & 77.27 & 76.51 & 77.23 & Yes & $0^{*}$ & $0.72^{*}$ & & & $76.55^{*}$ & $77.27^{*}$ & & \\
\hline $9 \mathrm{H}-6$ & 1.42 & 77.27 & 78.69 & 77.23 & 78.64 & Yes & $0^{*}$ & $1.42^{*}$ & & & $77.27^{*}$ & $78.69^{*}$ & & \\
\hline $9 \mathrm{H}-7$ & 0.65 & 78.69 & 79.34 & 78.64 & 79.28 & Yes & $0^{*}$ & $0.65^{*}$ & & & $78.69^{*}$ & $79.34^{*}$ & & \\
\hline 9H-CC & 0.12 & 79.34 & 79.46 & 79.28 & 79.4 & Yes & $0^{*}$ & $0.12^{*}$ & & & $79.34^{*}$ & $79.46^{*}$ & & \\
\hline $10 \mathrm{H}-1$ & 1.5 & 79.4 & 80.9 & 79.4 & 80.9 & Yes & & & $0^{\dagger}$ & $0.02^{\dagger}$ & & & $79.4^{\dagger}$ & $79.42^{\dagger}$ \\
\hline $11 \mathrm{H}-6$ & 1.42 & 95.2 & 96.62 & 95.14 & 96.55 & Yes & $0.44^{*}$ & $1.42^{*}$ & & & $95.64^{*}$ & $96.62^{*}$ & & \\
\hline $11 \mathrm{H}-\mathrm{CC}$ & 0.15 & 96.62 & 96.77 & 96.55 & 96.7 & Yes & $0^{*}$ & $0.15^{*}$ & & & $96.62^{*}$ & $96.77^{*}$ & & \\
\hline $14 \mathrm{H}-5$ & 1 & 118.43 & 119.43 & 118.42 & 119.41 & Yes & $0.53^{*}$ & $1^{*}$ & & & $118.96^{*}$ & $119.43^{*}$ & & \\
\hline $14 \mathrm{H}-6$ & 0.74 & 119.43 & 120.17 & 119.41 & 120.15 & Yes & $0^{*}$ & $0.74^{*}$ & & & $119.43^{*}$ & $120.17^{*}$ & & \\
\hline $14 \mathrm{H}-\mathrm{CC}$ & 0.15 & 120.17 & 120.32 & 120.15 & 120.3 & Yes & $0^{*}$ & $0.15^{*}$ & & & $120.17^{*}$ & $120.32^{*}$ & & \\
\hline $15 \mathrm{H}-1$ & 1.52 & 120.3 & 121.82 & 120.3 & 121.63 & Yes & & & $0.5^{*}$ & $1.52^{*}$ & & & $120.8^{*}$ & $121.82^{*}$ \\
\hline $15 \mathrm{H}-2$ & 1.28 & 121.82 & 123.1 & 121.63 & 122.75 & Yes & $0.66^{*}$ & $1.28^{*}$ & $0^{*}$ & $0.66^{*}$ & $122.48^{*}$ & $123.1^{*}$ & $121.82^{*}$ & $122.48^{*}$ \\
\hline $15 \mathrm{H}-3$ & 1.5 & 123.1 & 124.6 & 122.75 & 124.06 & Yes & $0^{*}$ & $1.5^{*}$ & & & $123.1^{*}$ & $124.6^{*}$ & & \\
\hline $15 \mathrm{H}-\mathrm{CC}$ & 0.04 & 124.6 & 124.64 & 124.06 & 124.1 & Yes & $0^{*}$ & $0.04^{*}$ & & & $124.6^{*}$ & $124.64^{*}$ & & \\
\hline $340-U 1396$ & & & & & & & & & & & & & & \\
\hline $8 \mathrm{H}-1$ & 1.51 & 62.6 & 64.11 & 62.6 & 64.02 & No & & & $0^{\dagger}$ & $0.03^{\dagger}$ & & & $62.6^{\dagger}$ & $62.63^{\dagger}$ \\
\hline $12 \mathrm{H}-5$ & 0.88 & 106.36 & 107.24 & 106.29 & 107.16 & Yes & $0.46^{*}$ & $0.88^{*}$ & & & $106.82^{*}$ & $107.24^{*}$ & & \\
\hline $12 \mathrm{H}-\mathrm{CC}$ & 0.14 & 107.24 & 107.38 & 107.16 & 107.3 & Yes & $0^{*}$ & $0.14^{*}$ & & & $107.24^{*}$ & $107.38^{*}$ & & \\
\hline $13 \mathrm{H}-1$ & 1.5 & 107.3 & 108.8 & 107.3 & 108.79 & No & & & $0^{\dagger}$ & $0.05^{\dagger}$ & & & $107.3^{\dagger}$ & $107.35^{\dagger}$ \\
\hline $13 \mathrm{H}-6$ & 1.01 & 114.8 & 115.81 & 114.76 & 115.76 & No & $0.79^{*}$ & $1.01^{*}$ & & & $115.59^{*}$ & 115.81* & & \\
\hline $13 \mathrm{H}-\mathrm{CC}$ & 0.14 & 115.81 & 115.95 & 115.76 & 115.9 & No & $0^{*}$ & $0.14^{*}$ & & & $115.81^{*}$ & $115.95^{*}$ & & \\
\hline $14 \mathrm{H}-1$ & 1.5 & 115.9 & 117.4 & 115.9 & 117.33 & No & & & $0^{\dagger}$ & $0.06^{\dagger}$ & & & $115.9^{\dagger}$ & $115.96^{\dagger}$ \\
\hline $340-U 139$ & & & & & & & & & & & & & & \\
\hline $2 \mathrm{H}-1$ & 1.5 & 5 & 6.5 & 5 & 5 & No & & & $0^{\dagger}$ & $0.03^{\dagger}$ & & & $5^{\dagger}$ & $5.03^{\dagger}$ \\
\hline $340-U 1396$ & & & & & & & & & & & & & & \\
\hline $6 \mathrm{H}-1$ & 1.5 & 46.4 & 47.9 & 46.4 & 47.82 & No & & & $0^{\dagger}$ & $0.06^{\dagger}$ & & & $46.4^{\dagger}$ & $46.46^{\dagger}$ \\
\hline $13 \mathrm{H}-3$ & 0.58 & 115.91 & 116.49 & 115.87 & 116.45 & Yes & $0.25^{*}$ & $1.51^{*}$ & & & $116.16^{*}$ & $117.42^{*}$ & & \\
\hline $13 \mathrm{H}-4$ & 0.56 & 116.49 & 117.05 & 116.45 & 117 & Yes & $0^{*}$ & $0.58^{*}$ & & & $116.49^{*}$ & $117.07^{*}$ & & \\
\hline $13 \mathrm{H}-5$ & 1.14 & 117.05 & 118.19 & 117 & 118.13 & Yes & $0^{*}$ & $0.56^{*}$ & & & $117.05^{*}$ & $117.61^{*}$ & & \\
\hline
\end{tabular}


Table T1 (continued). (Continued on next page.)

\begin{tabular}{|c|c|c|c|c|c|c|c|c|c|c|c|c|c|c|}
\hline \multirow{2}{*}{$\begin{array}{l}\text { Core, } \\
\text { section }\end{array}$} & \multirow{2}{*}{$\begin{array}{l}\text { Recovered } \\
\text { length } \\
(\mathrm{m})\end{array}$} & \multicolumn{2}{|c|}{ Depth CSF-A (m) } & \multicolumn{2}{|c|}{ Depth CSF-B (m) } & \multirow{2}{*}{$\begin{array}{l}\text { Partial } \\
\text { stroke }\end{array}$} & \multicolumn{2}{|c|}{$\begin{array}{c}\text { Fall-in* or flow-in }{ }^{\dagger} \\
\text { depth in section }(\mathrm{m})\end{array}$} & \multicolumn{2}{|c|}{$\begin{array}{l}\text { Possible fall-in* or } \\
\text { flow-in }{ }^{\dagger} \text { depth } \\
\text { in section }(\mathrm{m})\end{array}$} & \multicolumn{2}{|c|}{$\begin{array}{l}\text { Fall-in* or flow-in }^{\dagger} \\
\text { depth in hole } \\
\text { CSF-A }(m)\end{array}$} & $\begin{array}{c}\text { Possible } \mathrm{f} \\
\text { flow-in }^{\dagger} \\
\text { in hole C }\end{array}$ & $\begin{array}{l}\text { fall-in* or } \\
{ }^{\dagger} \text { depth } \\
\text { SF-A (m) }\end{array}$ \\
\hline & & Top & Bottom & Top & Bottom & & Top & Bottom & Top & Bottom & Top & Bottom & Top & Bottom \\
\hline $13 \mathrm{H}-6$ & 1.5 & 118.19 & 119.69 & 118.13 & 119.61 & Yes & $0^{*}$ & $1.14^{*}$ & & & 118.19* & $119.33^{*}$ & & \\
\hline $13 \mathrm{H}-7$ & 0.67 & 119.69 & 120.36 & 119.61 & 120.27 & Yes & $0^{*}$ & $0.67^{*}$ & & & 119.69* & $120.36^{*}$ & & \\
\hline $13 \mathrm{H}-\mathrm{CC}$ & 0.13 & 120.36 & 120.49 & 120.27 & 120.4 & Yes & $0^{*}$ & $0.13^{*}$ & & & $120.36^{*}$ & $120.49^{*}$ & & \\
\hline $14 \mathrm{H}-1$ & 1.49 & 120.4 & 121.89 & 120.4 & 121.86 & No & $0^{\dagger}$ & $0.02^{\dagger}$ & & & & & & \\
\hline 340-U139 & & & & & & & & & & & & & & \\
\hline $2 \mathrm{H}-\mathrm{CC}$ & 0.15 & 9.24 & 9.39 & 9.24 & 9.39 & No & & & $0^{*}$ & $0.15^{*}$ & & & $9.24^{*}$ & $9.39^{*}$ \\
\hline $4 \mathrm{H}-1$ & 1.5 & 19.3 & 20.8 & 19.3 & 20.76 & No & & & $0^{\dagger}$ & $0.17^{\dagger}$ & & & $19.3^{\dagger}$ & $19.47^{\dagger}$ \\
\hline $4 \mathrm{H}-7$ & 0.59 & 28.3 & 28.89 & 28.06 & 28.64 & No & & & $0.08^{*}$ & $0.59^{*}$ & & & $28.38^{*}$ & $28.89^{*}$ \\
\hline $4 \mathrm{H}-\mathrm{CC}$ & 0.17 & 28.89 & 29.06 & 28.64 & 28.8 & No & $0^{*}$ & $0.17^{*}$ & & & $28.89^{*}$ & $29.06^{*}$ & & \\
\hline $5 \mathrm{H}-1$ & 1.5 & 28.8 & 30.3 & 28.8 & 30.28 & Yes & & & $0^{\dagger}$ & $1.24^{\dagger}$ & & & $28.8^{\dagger}$ & $30.04^{\dagger}$ \\
\hline $5 \mathrm{H}-5$ & 1.1 & 34.8 & 35.9 & 34.74 & 35.83 & Yes & & & $0.41^{*}$ & $1.1^{*}$ & & & $35.21^{*}$ & $35.9^{\star}$ \\
\hline $5 \mathrm{H}-6$ & 0.6 & 35.9 & 36.5 & 35.83 & 36.42 & Yes & & & $0^{*}$ & $0.6^{*}$ & & & $35.9^{*}$ & $36.5^{*}$ \\
\hline $5 \mathrm{H}-\mathrm{CC}$ & 0.08 & 36.5 & 36.58 & 36.42 & 36.5 & Yes & & & $0^{*}$ & $0.08^{*}$ & & & $36.5^{*}$ & $36.58^{*}$ \\
\hline $6 \mathrm{H}-1$ & 1.5 & 36.5 & 38 & 36.5 & 37.98 & No & & & $0^{\dagger}$ & $0.25^{\dagger}$ & & & $36.5^{\dagger}$ & $36.75^{\dagger}$ \\
\hline $7 \mathrm{H}-1$ & 1.5 & 46 & 47.5 & 46 & 47.5 & Yes & & & $0^{\dagger}$ & $0.02^{\dagger}$ & & & $46^{\dagger}$ & $46.02^{\dagger}$ \\
\hline $7 \mathrm{H}-6$ & 1.5 & 52.61 & 54.11 & 52.6 & 54.09 & Yes & $0^{*}$ & $1.5^{*}$ & & & $52.61^{*}$ & $54.11^{*}$ & & \\
\hline $7 \mathrm{H}-7$ & 0.75 & 54.11 & 54.86 & 54.09 & 54.84 & Yes & $0^{*}$ & $0.75^{*}$ & & & $54.11^{*}$ & $54.86^{*}$ & & \\
\hline $8 \mathrm{H}-1$ & 0.93 & 55 & 55.93 & 55 & 55.92 & Yes & $0^{*}$ & $0.93^{*}$ & & & $55^{*}$ & $55.93^{*}$ & & \\
\hline $8 \mathrm{H}-2$ & 0.93 & 55.93 & 56.86 & 55.92 & 56.84 & Yes & $0^{*}$ & $0.93^{*}$ & & & $55.93^{*}$ & $56.86^{*}$ & & \\
\hline $8 \mathrm{H}-3$ & 1.09 & 56.86 & 57.95 & 56.84 & 57.92 & Yes & $0^{*}$ & $1.09^{*}$ & & & $56.86^{*}$ & $57.95^{*}$ & & \\
\hline $8 \mathrm{H}-4$ & 0.98 & 57.95 & 58.93 & 57.92 & 58.9 & Yes & $0^{*}$ & $0.98^{*}$ & & & $57.95^{*}$ & $58.93^{*}$ & & \\
\hline $8 \mathrm{H}-5$ & 1.21 & 58.93 & 60.14 & 58.9 & 60.09 & Yes & $0^{*}$ & $1.21^{*}$ & & & $58.93^{*}$ & $60.14^{*}$ & & \\
\hline $8 \mathrm{H}-6$ & 1.36 & 60.14 & 61.5 & 60.09 & 61.44 & Yes & $0^{*}$ & $1.36^{*}$ & & & $60.14^{*}$ & $61.5^{*}$ & & \\
\hline $8 \mathrm{H}-\mathrm{CC}$ & 0.16 & 61.5 & 61.66 & 61.44 & 61.6 & Yes & $0^{*}$ & $0.16^{*}$ & & & $61.5^{*}$ & $61.66^{*}$ & & \\
\hline $9 \mathrm{H}-1$ & 1.07 & 61.6 & 62.67 & 61.6 & 62.66 & Yes & $0^{*}$ & $1.07^{*}$ & & & $61.6^{*}$ & $62.67^{*}$ & & \\
\hline $9 \mathrm{H}-2$ & 1.25 & 62.67 & 63.92 & 62.66 & 63.91 & Yes & $0^{*}$ & $1.25^{*}$ & & & $62.67^{*}$ & $63.92^{*}$ & & \\
\hline $9 \mathrm{H}-3$ & 1.5 & 63.92 & 65.42 & 63.91 & 65.4 & Yes & $0^{*}$ & $1.5^{*}$ & & & $63.92^{*}$ & $65.42^{*}$ & & \\
\hline $9 \mathrm{H}-4$ & 1.02 & 65.42 & 66.44 & 65.4 & 66.41 & Yes & $0^{*}$ & $1.02^{*}$ & & & $65.42^{*}$ & $66.44^{*}$ & & \\
\hline $9 \mathrm{H}-\mathrm{CC}$ & 0.19 & 66.44 & 66.63 & 66.41 & 66.6 & Yes & $0^{*}$ & $0.19^{*}$ & & & $66.44^{*}$ & $66.63^{*}$ & & \\
\hline $10 \mathrm{H}-1$ & 1.5 & 66.6 & 68.1 & 66.6 & 68.1 & Yes & $0^{\dagger}$ & $0.1^{\dagger}$ & $0.1^{\dagger}$ & $1.5^{\dagger}$ & $66.6^{\dagger}$ & $66.7^{\dagger}$ & $66.7^{\dagger}$ & $68.1^{\dagger}$ \\
\hline $10 \mathrm{H}-2$ & 1.5 & 68.1 & 69.6 & 68.1 & 69.59 & Yes & & & $0^{\dagger}$ & $0.49^{\dagger}$ & & & $68.1^{\dagger}$ & $68.59^{\dagger}$ \\
\hline $10 \mathrm{H}-4$ & 1.5 & 71.1 & 72.6 & 71.09 & 72.58 & Yes & & & $0.9^{*}$ & $1.5^{*}$ & & & $72^{*}$ & $72.6^{*}$ \\
\hline $10 \mathrm{H}-5$ & 0.67 & 72.6 & 73.27 & 72.58 & 73.25 & Yes & & & $0^{*}$ & $0.67^{*}$ & & & $72.6^{*}$ & $73.27^{*}$ \\
\hline $10 \mathrm{H}-\mathrm{CC}$ & 0.25 & 73.27 & 73.52 & 73.25 & 73.5 & Yes & & & $0^{*}$ & $0.25^{*}$ & & & $73.27^{*}$ & $73.52^{*}$ \\
\hline $11 \mathrm{H}-1$ & 1.51 & 73.5 & 75.01 & 73.5 & 75.01 & Yes & $0^{\dagger}$ & $0.3^{\dagger}$ & & & $73.5^{\dagger}$ & $73.8^{\dagger}$ & & \\
\hline $11 \mathrm{H}-5$ & 1.49 & 78.89 & 80.38 & 78.88 & 80.37 & Yes & $1.29^{*}$ & $1.49^{*}$ & & & $80.18^{*}$ & $80.38^{*}$ & & \\
\hline $11 \mathrm{H}-\mathrm{CC}$ & 0.13 & 80.38 & 80.51 & 80.37 & 80.5 & Yes & $0^{*}$ & $0.13^{*}$ & & & $80.38^{*}$ & $80.51^{*}$ & & \\
\hline $12 \mathrm{H}-2$ & 1.5 & 82.05 & 83.55 & 82.05 & 83.54 & Yes & $0^{*}$ & $1.5^{*}$ & & & $82.05^{*}$ & $83.55^{*}$ & & \\
\hline $12 \mathrm{H}-3$ & 1.5 & 83.55 & 85.05 & 83.54 & 85.04 & Yes & $0^{*}$ & $1.5^{*}$ & & & $83.55^{*}$ & $85.05^{*}$ & & \\
\hline $12 \mathrm{H}-4$ & 1.3 & 85.05 & 86.35 & 85.04 & 86.34 & Yes & $0^{*}$ & $1.3^{*}$ & & & $85.05^{*}$ & $86.35^{*}$ & & \\
\hline $12 \mathrm{H}-5$ & 0.6 & 86.35 & 86.95 & 86.34 & 86.94 & Yes & $0^{*}$ & $0.6^{*}$ & & & $86.35^{*}$ & $86.95^{*}$ & & \\
\hline $12 \mathrm{H}-\mathrm{CC}$ & 0.16 & 86.95 & 87.11 & 86.94 & 87.1 & Yes & $0^{*}$ & $0.16^{*}$ & & & $86.95^{\star}$ & $87.11^{*}$ & & \\
\hline $13 \mathrm{H}-1$ & 1.36 & 87.1 & 88.46 & 87.1 & 88.46 & Yes & $0^{\dagger}$ & $0.07^{\dagger}$ & & & $87.1^{\dagger}$ & $87.17^{\dagger}$ & & \\
\hline $13 \mathrm{H}-\mathrm{CC}$ & 0.13 & 94.87 & 95 & 94.87 & 95 & Yes & $0^{*}$ & $0.13^{*}$ & & & $94.87^{*}$ & $95^{*}$ & & \\
\hline $14 \mathrm{H}-1$ & 0.43 & 95 & 95.43 & 95 & 95.43 & Yes & $0^{\dagger}$ & $0.18^{\dagger}$ & & & $95^{\dagger}$ & $95.18^{\dagger}$ & & \\
\hline $14 \mathrm{H}-\mathrm{CC}$ & 0.11 & 101.84 & 101.95 & 101.79 & 101.9 & Yes & $0^{*}$ & $0.11^{*}$ & & & $101.84^{*}$ & $101.95^{*}$ & & \\
\hline $15 \mathrm{H}-\mathrm{CC}$ & 0.16 & 101.9 & 102.06 & 101.9 & 102.06 & Yes & $0^{*}$ & $0.16^{*}$ & & & $101.9^{*}$ & $102.06^{*}$ & & \\
\hline $17 \mathrm{H}-1$ & 0.59 & 106.5 & 107.09 & 106.5 & 107.09 & Yes & $0^{\dagger}$ & $0.59^{\dagger}$ & & & $106.5^{\dagger}$ & $107.09^{\dagger}$ & & \\
\hline $17 \mathrm{H}-2$ & 1.5 & 107.09 & 108.59 & 107.09 & 108.59 & Yes & $0^{*}$ & $1.5^{*}$ & & & 107.09* & $108.59^{*}$ & & \\
\hline $17 \mathrm{H}-3$ & 1.23 & 108.59 & 109.82 & 108.59 & 109.82 & Yes & $0^{*}$ & $1.23^{*}$ & & & $108.59^{*}$ & $109.82^{*}$ & & \\
\hline $17 \mathrm{H}-\mathrm{CC}$ & 0.17 & 109.82 & 109.99 & 109.82 & 109.99 & Yes & $0^{*}$ & $0.17^{*}$ & & & $109.82^{*}$ & 109.99* & & \\
\hline $18 \mathrm{H}-1$ & 1.5 & 110 & 111.5 & 110 & 111.5 & Yes & $0^{\dagger}$ & $0.57^{\dagger}$ & & & $110^{\dagger}$ & $110.57^{\dagger}$ & & \\
\hline $18 \mathrm{H}-5$ & 1.25 & 114.92 & 116.17 & 114.92 & 116.17 & Yes & & & $0.8^{*}$ & $1.25^{\star}$ & & & $115.72^{*}$ & $116.17^{*}$ \\
\hline $18 \mathrm{H}-\mathrm{CC}$ & 0.13 & 116.17 & 116.3 & 116.17 & 116.3 & Yes & & & $0^{*}$ & $0.13^{*}$ & & & $116.17^{*}$ & $116.3^{*}$ \\
\hline $19 \mathrm{H}-1$ & 1.5 & 116.3 & 117.8 & 116.3 & 117.79 & Yes & & & $0^{\dagger}$ & $0.16^{\dagger}$ & & & $116.3^{\dagger}$ & $116.46^{\dagger}$ \\
\hline $19 \mathrm{H}-2$ & 1.5 & 117.8 & 119.3 & 117.79 & 119.27 & Yes & & & $0.24^{\star}$ & $1.5^{*}$ & & & $118.04^{*}$ & $119.3^{*}$ \\
\hline $19 \mathrm{H}-3$ & 1.5 & 119.3 & 120.8 & 119.27 & 120.76 & Yes & & & $0^{*}$ & $1.5^{*}$ & & & $119.3^{*}$ & $120.8^{*}$ \\
\hline $19 \mathrm{H}-4$ & 0.55 & 120.8 & 121.35 & 120.76 & 121.3 & Yes & & & $0^{*}$ & $0.55^{*}$ & & & $120.8^{*}$ & $121.35^{*}$ \\
\hline $19 \mathrm{H}-\mathrm{CC}$ & 0.2 & 121.35 & 121.55 & 121.3 & 121.5 & Yes & & & $0^{*}$ & $0.07^{\star}$ & & & $121.35^{*}$ & $121.42^{*}$ \\
\hline $20 \mathrm{H}-\mathrm{CC}$ & 0.25 & 121.5 & 121.75 & 121.5 & 121.7 & Yes & $0.07^{*}$ & $0.15^{*}$ & & & $121.57^{*}$ & $121.65^{*}$ & & \\
\hline 340-U1397 & & & & & & & & & & & & & & \\
\hline $4 \mathrm{H}-5$ & 1.5 & 31.6 & 33.1 & 31.54 & 33.03 & Yes & $1.04^{*}$ & $1.5^{*}$ & & & $32.64^{*}$ & $33.1^{*}$ & & \\
\hline $4 \mathrm{H}-6$ & 0.94 & 33.1 & 34.04 & 33.03 & 33.96 & Yes & $0^{*}$ & $0.94^{*}$ & & & $33.1^{*}$ & $34.04^{*}$ & & \\
\hline $4 \mathrm{H}-\mathrm{CC}$ & 0.14 & 34.04 & 34.18 & 33.96 & 34.1 & Yes & $0^{*}$ & $0.14^{*}$ & & & $34.04^{*}$ & $34.18^{*}$ & & \\
\hline $5 \mathrm{H}-7$ & 0.59 & 42.65 & 43.24 & 42.65 & 43.24 & No & $0.45^{*}$ & $0.59^{\star}$ & & & $43.1^{*}$ & $43.24^{*}$ & & \\
\hline $5 \mathrm{H}-\mathrm{CC}$ & 0.11 & 43.24 & 43.35 & 43.24 & 43.35 & No & $0^{*}$ & $0.11^{*}$ & & & $43.24^{*}$ & $43.35^{*}$ & & \\
\hline
\end{tabular}


Table T1 (continued). (Continued on next page.)

\begin{tabular}{|c|c|c|c|c|c|c|c|c|c|c|c|c|c|c|}
\hline \multirow{2}{*}{$\begin{array}{l}\text { Core, } \\
\text { section }\end{array}$} & \multirow{2}{*}{$\begin{array}{l}\text { Recovered } \\
\text { length } \\
(\mathrm{m})\end{array}$} & \multicolumn{2}{|c|}{ Depth CSF-A (m) } & \multicolumn{2}{|c|}{ Depth CSF-B (m) } & \multirow{2}{*}{$\begin{array}{l}\text { Partial } \\
\text { stroke }\end{array}$} & \multicolumn{2}{|c|}{$\begin{array}{c}\text { Fall-in* or flow-in }{ }^{\dagger} \\
\text { depth in section }(\mathrm{m})\end{array}$} & \multicolumn{2}{|c|}{$\begin{array}{l}\text { Possible fall-in* or } \\
\text { flow-in }{ }^{\dagger} \text { depth } \\
\text { in section }(\mathrm{m})\end{array}$} & $\begin{array}{r}\text { Fall-in* or } \\
\text { depth i } \\
\text { CSF-A } \\
\end{array}$ & $\begin{array}{l}\text { flow-in }^{\dagger} \\
\text { n hole } \\
\text { (m) }\end{array}$ & $\begin{array}{r}\text { Possible f } \\
\text { flow-in } \\
\text { in hole C } \\
\end{array}$ & $\begin{array}{l}\text { all-in* or } \\
\text { depth } \\
\text { SF-A (m) }\end{array}$ \\
\hline & & Top & Bottom & Top & Bottom & & Top & Bottom & Top & Bottom & Top & Bottom & Top & Bottom \\
\hline $6 \mathrm{H}-\mathrm{CC}$ & 0.1 & 53 & 53.1 & 53 & 53.1 & No & $0^{*}$ & $0.1^{*}$ & & & $53^{*}$ & $53.1^{*}$ & & \\
\hline $7 \mathrm{H}-1$ & 1.5 & 53.1 & 54.6 & 53.1 & 54.58 & Yes & $0.42^{*}$ & $1.5^{*}$ & & & $53.52^{*}$ & $54.6^{*}$ & & \\
\hline $7 \mathrm{H}-2$ & 1.33 & 54.6 & 55.93 & 54.58 & 55.9 & Yes & $0^{*}$ & $1.33^{*}$ & & & $54.6^{*}$ & $55.93^{*}$ & & \\
\hline $7 \mathrm{H}-6$ & 1.21 & 59.42 & 60.63 & 59.36 & 60.56 & Yes & $0^{*}$ & $1.21^{*}$ & & & $59.42^{*}$ & $60.63^{*}$ & & \\
\hline $7 \mathrm{H}-7$ & 0.6 & 60.63 & 61.23 & 60.56 & 61.15 & Yes & $0^{*}$ & $0.6^{*}$ & & & $60.63^{*}$ & $61.23^{*}$ & & \\
\hline $7 \mathrm{H}-\mathrm{CC}$ & 0.15 & 61.23 & 61.38 & 61.15 & 61.3 & Yes & $0^{*}$ & $0.15^{*}$ & & & $61.23^{*}$ & $61.38^{*}$ & & \\
\hline $8 \mathrm{H}-1$ & 1.5 & 61.3 & 62.8 & 61.3 & 62.79 & Yes & $0^{\dagger}$ & $1.5^{\dagger}$ & & & $61.3^{\dagger}$ & $62.8^{\dagger}$ & & \\
\hline $8 \mathrm{H}-2$ & 1.5 & 62.8 & 64.3 & 62.79 & 64.28 & Yes & $0^{\dagger}$ & $1.25^{\dagger}$ & & & $62.8^{\dagger}$ & $64.05^{\dagger}$ & & \\
\hline $8 \mathrm{H}-3$ & 1.12 & 64.3 & 65.42 & 64.28 & 65.38 & Yes & $0.88^{*}$ & $1.12^{*}$ & & & $65.18^{*}$ & $65.42^{*}$ & & \\
\hline $10 \mathrm{H}-4$ & 1.15 & 83.1 & 84.25 & 83.08 & 84.23 & Yes & $0.43^{*}$ & $1.15^{*}$ & & & $83.53^{*}$ & $84.25^{*}$ & & \\
\hline $10 \mathrm{H}-5$ & 1.5 & 84.25 & 85.75 & 84.23 & 85.72 & Yes & $0^{*}$ & $1.5^{*}$ & & & $84.25^{*}$ & $85.75^{*}$ & & \\
\hline $10 \mathrm{H}-6$ & 1.31 & 85.75 & 87.06 & 85.72 & 87.02 & Yes & $0^{*}$ & $1.31^{*}$ & & & $85.75^{*}$ & $87.06^{*}$ & & \\
\hline $10 \mathrm{H}-7$ & 0.64 & 87.06 & 87.7 & 87.02 & 87.66 & Yes & $0^{*}$ & $0.64^{*}$ & & & $87.06^{*}$ & $87.7^{*}$ & & \\
\hline $10 \mathrm{H}-\mathrm{CC}$ & 0.24 & 87.7 & 87.94 & 87.66 & 87.9 & Yes & $0^{*}$ & $0.24^{*}$ & & & $87.7^{*}$ & $87.94^{*}$ & & \\
\hline $11 \mathrm{H}-1$ & 1.23 & 87.9 & 89.13 & 87.9 & 89.11 & Yes & $0^{*}$ & $1.23^{*}$ & & & $87.9^{*}$ & $89.13^{*}$ & & \\
\hline $11 \mathrm{H}-2$ & 1.5 & 89.13 & 90.63 & 89.11 & 90.59 & Yes & $0^{*}$ & $1.5^{*}$ & & & $89.13^{*}$ & $90.63^{*}$ & & \\
\hline $11 \mathrm{H}-3$ & 0.89 & 90.63 & 91.52 & 90.59 & 91.46 & Yes & $0^{*}$ & $0.89^{*}$ & & & $90.63^{*}$ & $91.52^{*}$ & & \\
\hline $11 \mathrm{H}-\mathrm{CC}$ & 0.24 & 91.52 & 91.76 & 91.46 & 91.7 & Yes & $0^{*}$ & $0.24^{\star}$ & & & $91.52^{*}$ & $91.76^{*}$ & & \\
\hline $12 \mathrm{H}-1$ & 1.5 & 91.7 & 93.2 & 91.7 & 93.2 & Yes & $0^{\dagger}$ & $0.15^{\dagger}$ & & & $91.7^{\dagger}$ & $91.85^{\dagger}$ & & \\
\hline $12 \mathrm{H}-3$ & 1.5 & 94.7 & 96.2 & 94.7 & 96.2 & Yes & $0.14^{*}$ & $1.5^{*}$ & & & $94.84^{*}$ & $96.2^{\star}$ & & \\
\hline $12 \mathrm{H}-4$ & 1.21 & 96.2 & 97.41 & 96.2 & 97.41 & Yes & $0^{*}$ & $1.21^{*}$ & & & $96.2^{*}$ & $97.41^{*}$ & & \\
\hline $12 \mathrm{H}-5$ & 0.67 & 97.41 & 98.08 & 97.41 & 98.08 & Yes & $0^{*}$ & $0.67^{*}$ & & & $97.41^{*}$ & $98.08^{*}$ & & \\
\hline $12 \mathrm{H}-\mathrm{CC}$ & 0.1 & 98.08 & 98.18 & 98.08 & 98.18 & Yes & $0^{*}$ & $0.1^{*}$ & & & $98.08^{*}$ & $98.18^{*}$ & & \\
\hline $17 \mathrm{H}-1$ & 0.26 & 113 & 113.26 & 113 & 113.2 & Yes & $0^{\dagger}$ & $0.26^{\dagger}$ & & & $113^{\dagger}$ & $113.26^{\dagger}$ & & \\
\hline $340-U 139$ & & & & & & & & & & & & & & \\
\hline $1 \mathrm{H}-1$ & 1.5 & 0 & 1.5 & 0 & 1.47 & No & $1^{*}$ & $1.5^{*}$ & & & $1^{*}$ & $1.5^{*}$ & & \\
\hline $1 \mathrm{H}-2$ & 1.08 & 1.5 & 2.58 & 1.47 & 2.52 & No & $0^{*}$ & $1.08^{*}$ & & & $1.5^{*}$ & $2.58^{*}$ & & \\
\hline $1 \mathrm{H}-\mathrm{CC}$ & 0.18 & 2.58 & 2.76 & 2.52 & 2.7 & No & $0^{*}$ & $0.18^{*}$ & & & $2.58^{*}$ & $2.76^{*}$ & & \\
\hline $2 \mathrm{H}-1$ & 1.5 & 2.7 & 4.2 & 2.7 & 4.2 & No & $0^{*}$ & $1.5^{*}$ & & & $2.7^{*}$ & $4.2^{*}$ & & \\
\hline $2 \mathrm{H}-2$ & 1.5 & 4.2 & 5.7 & 4.2 & 5.7 & No & $0^{*}$ & $1.5^{*}$ & & & $4.2^{*}$ & $5.7^{*}$ & & \\
\hline $2 \mathrm{H}-3$ & 1.5 & 5.7 & 7.2 & 5.7 & 7.2 & No & $0^{*}$ & $1.5^{*}$ & & & $5.7^{*}$ & $7.2^{*}$ & & \\
\hline $2 \mathrm{H}-4$ & 1.5 & 7.2 & 8.7 & 7.2 & 8.7 & No & $0^{*}$ & $1.5^{*}$ & & & $7.2^{*}$ & $8.7^{*}$ & & \\
\hline $2 \mathrm{H}-5$ & 1.09 & 8.7 & 9.79 & 8.7 & 9.79 & No & $0^{*}$ & $1.09^{*}$ & & & $8.7^{*}$ & $9.79^{\star}$ & & \\
\hline $2 \mathrm{H}-6$ & 1.54 & 9.79 & 11.33 & 9.79 & 11.33 & No & $0^{*}$ & $1.54^{*}$ & & & $9.79 *$ & $11.33^{*}$ & & \\
\hline $2 \mathrm{H}-\mathrm{CC}$ & 0.18 & 11.33 & 11.51 & 11.33 & 11.51 & No & $0^{*}$ & $0.18^{*}$ & & & $11.33^{*}$ & $11.51^{*}$ & & \\
\hline $5 \mathrm{H}-1$ & 1.5 & 31.5 & 33 & 31.5 & 32.99 & Yes & $0^{\dagger}$ & $1.29^{\dagger}$ & & & $31.5^{\dagger}$ & $32.79^{\dagger}$ & & \\
\hline $5 \mathrm{H}-6$ & 0.8 & 37.87 & 38.67 & 37.82 & 38.61 & Yes & $0.65^{\star}$ & $0.8^{*}$ & & & $38.52^{*}$ & $38.67^{*}$ & & \\
\hline $5 \mathrm{H}-\mathrm{CC}$ & 0.09 & 38.67 & 38.76 & 38.61 & 38.7 & Yes & $0^{*}$ & $0.09^{*}$ & & & $38.67^{*}$ & $38.76^{*}$ & & \\
\hline $6 \mathrm{H}-1$ & 1.52 & 38.7 & 40.22 & 38.7 & 40.19 & Yes & $0^{\dagger}$ & $0.6^{\dagger}$ & & & $38.7^{\dagger}$ & $39.3^{\dagger}$ & & \\
\hline $7 \mathrm{H}-4$ & 0.85 & 50.1 & 50.95 & 50.04 & 50.88 & Yes & & & $0.42^{*}$ & $0.85^{*}$ & & & $50.52^{*}$ & $50.95^{*}$ \\
\hline $7 \mathrm{H}-\mathrm{CC}$ & 0.02 & 50.95 & 50.97 & 50.88 & 50.9 & Yes & & & $0^{*}$ & $0.02^{*}$ & & & $50.95^{*}$ & $50.97^{*}$ \\
\hline $8 \mathrm{H}-1$ & 1.5 & 50.9 & 52.4 & 50.9 & 52.34 & No & & & $0^{\dagger}$ & $1.5^{\dagger}$ & & & $50.9^{\dagger}$ & $52.4^{\dagger}$ \\
\hline $8 \mathrm{H}-2$ & 1.5 & 52.4 & 53.9 & 52.34 & 53.78 & No & & & $0^{\dagger}$ & $1.5^{\dagger}$ & & & $52.4^{\dagger}$ & $53.9^{\dagger}$ \\
\hline $8 \mathrm{H}-3$ & 1.5 & 53.9 & 55.4 & 53.78 & 55.21 & No & & & $0^{\dagger}$ & $1.5^{\dagger}$ & & & $53.9^{\dagger}$ & $55.4^{\dagger}$ \\
\hline $8 \mathrm{H}-4$ & 1.5 & 55.4 & 56.9 & 55.21 & 56.65 & No & & & $0^{\dagger}$ & $1.5^{\dagger}$ & & & $55.4^{\dagger}$ & $56.5^{\dagger}$ \\
\hline $9 \mathrm{H}-6$ & 1.31 & 67.84 & 69.15 & 67.84 & 69.15 & No & & & $1.2^{*}$ & $1.31^{*}$ & & & $69.04^{*}$ & $69.15^{*}$ \\
\hline $9 \mathrm{H}-\mathrm{CC}$ & 0.03 & 69.15 & 69.18 & 69.15 & 69.18 & No & & & $0^{*}$ & $0.03^{*}$ & & & $69.15^{*}$ & $69.18^{*}$ \\
\hline $11 \mathrm{H}-1$ & 1.5 & 79.4 & 80.9 & 79.4 & 80.83 & No & & & $0^{\dagger}$ & $0.25^{\dagger}$ & & & $79.4^{\dagger}$ & $79.65^{\dagger}$ \\
\hline $12 \mathrm{H}-4$ & 0.79 & 93.33 & 94.12 & 93.28 & 94.06 & Yes & $0.75^{\star}$ & $0.79^{\star}$ & & & $94.08^{*}$ & $94.12^{*}$ & & \\
\hline
\end{tabular}


Table T1 (continued). (Continued on next page.)

\begin{tabular}{|c|c|c|c|c|c|c|c|c|c|c|c|c|c|c|}
\hline \multirow{2}{*}{$\begin{array}{l}\text { Core, } \\
\text { section }\end{array}$} & \multirow{2}{*}{$\begin{array}{l}\text { Recovered } \\
\text { length } \\
(\mathrm{m})\end{array}$} & \multicolumn{2}{|c|}{ Depth CSF-A (m) } & \multicolumn{2}{|c|}{ Depth CSF-B (m) } & \multirow{2}{*}{$\begin{array}{l}\text { Partial } \\
\text { stroke }\end{array}$} & \multicolumn{2}{|c|}{$\begin{array}{c}\text { Fall-in* or flow-in } \\
\text { depth in section }(\mathrm{m}) \\
\end{array}$} & \multicolumn{2}{|c|}{$\begin{array}{l}\text { Possible fall-in* or } \\
\text { flow-in }{ }^{\dagger} \text { depth } \\
\text { in section }(\mathrm{m}) \\
\end{array}$} & $\begin{array}{r}\text { Fall-in* or } \\
\text { depth } \\
\text { CSF-A }\end{array}$ & $\begin{array}{l}r \text { flow-in } \\
\text { in hole } \\
\mathrm{A}(\mathrm{m})\end{array}$ & $\begin{array}{r}\text { Possible f } \\
\text { flow-in } \\
\text { in hole C } \\
\end{array}$ & $\begin{array}{l}\text { all-in* or } \\
\text { depth } \\
\text { SF-A (m) }\end{array}$ \\
\hline & & Top & Bottom & Top & Bottom & & Top & Bottom & Top & Bottom & Top & Bottom & Top & Bottom \\
\hline $12 \mathrm{H}-5$ & 0.58 & 94.12 & 94.7 & 94.06 & 94.63 & Yes & $0^{*}$ & $0.58^{*}$ & & & $94.12^{*}$ & $94.7^{*}$ & & \\
\hline $12 \mathrm{H}-6$ & 1.36 & 94.7 & 96.06 & 94.63 & 95.97 & Yes & $0^{*}$ & $1.36^{*}$ & & & $94.7^{*}$ & $96.06^{*}$ & & \\
\hline $12 \mathrm{H}-\mathrm{CC}$ & 0.13 & 96.06 & 96.19 & 95.97 & 96.1 & Yes & $0^{*}$ & $0.13^{*}$ & & & $96.06^{*}$ & $96.19^{*}$ & & \\
\hline $13 \mathrm{H}-1$ & 1.5 & 96.1 & 97.6 & 96.1 & 97.57 & No & & & $0^{\dagger}$ & $1.5^{\dagger}$ & & & $96.1^{\dagger}$ & $97.6^{\dagger}$ \\
\hline $13 \mathrm{H}-2$ & 1.5 & 97.6 & 99.1 & 97.57 & 99.04 & No & & & $0^{\dagger}$ & $0.28^{\dagger}$ & & & $97.6^{\dagger}$ & $97.88^{\dagger}$ \\
\hline 340-U139 & & & & & & & & & & & & & & \\
\hline $1 \mathrm{H}-1$ & 1.5 & 0 & 1.5 & 0 & 1.48 & No & $0.69^{*}$ & $1.5^{*}$ & & & $0.69^{*}$ & $1.5^{*}$ & & \\
\hline $1 \mathrm{H}-2$ & 1.5 & 1.5 & 3 & 1.48 & 2.97 & No & $0^{*}$ & $1.5^{*}$ & & & $1.5^{*}$ & $3^{*}$ & & \\
\hline $1 \mathrm{H}-3$ & 1.5 & 3 & 4.5 & 2.97 & 4.45 & No & $0^{*}$ & $1.5^{*}$ & & & $3^{*}$ & $4.5^{*}$ & & \\
\hline $1 \mathrm{H}-4$ & 1.5 & 4.5 & 6 & 4.45 & 5.93 & No & $0^{*}$ & $1.5^{*}$ & & & $4.5^{*}$ & $6^{*}$ & & \\
\hline $1 \mathrm{H}-5$ & 1.21 & 6 & 7.21 & 5.93 & 7.13 & No & $0^{*}$ & $1.21^{*}$ & & & $6^{*}$ & 7.21 & & \\
\hline $1 \mathrm{H}-6$ & 0.58 & 7.21 & 7.79 & 7.13 & 7.7 & No & $0^{*}$ & $0.58^{*}$ & & & $7.21^{*}$ & $7.79^{*}$ & & \\
\hline $1 \mathrm{H}-\mathrm{CC}$ & 0.2 & 7.79 & 7.99 & 7.7 & 7.9 & No & $0^{*}$ & $0.2^{*}$ & & & $7.79^{*}$ & $7.99^{*}$ & & \\
\hline $2 \mathrm{H}-1$ & 1.39 & 7.9 & 9.29 & 7.9 & 9.27 & Yes & $0^{\dagger}$ & $0.44^{\dagger}$ & $0^{\dagger}$ & $1.39^{\dagger}$ & $7.9^{\dagger}$ & $8.34^{\dagger}$ & $8.34^{\dagger}$ & $9.29^{\dagger}$ \\
\hline $2 \mathrm{H}-2$ & 1.4 & 9.29 & 10.69 & 9.27 & 10.66 & Yes & & & $0^{\dagger}$ & $1.4^{\dagger}$ & & & $9.29^{\dagger}$ & $10.69^{\dagger}$ \\
\hline $2 \mathrm{H}-3$ & 1.23 & 10.69 & 11.92 & 10.66 & 11.87 & Yes & $1.17^{*}$ & $1.23^{*}$ & $0^{\dagger}$ & $0.76^{\dagger}$ & $11.86^{*}$ & $11.92^{*}$ & $10.69^{\dagger}$ & $11.45^{\dagger}$ \\
\hline $2 \mathrm{H}-4$ & 0.82 & 11.92 & 12.74 & 11.87 & 12.68 & Yes & $0^{*}$ & $0.82^{*}$ & & & $11.92^{*}$ & $12.74^{*}$ & & \\
\hline $2 \mathrm{H}-5$ & 1.29 & 12.74 & 14.03 & 12.68 & 13.96 & Yes & $0^{*}$ & $1.29^{*}$ & & & $12.74^{*}$ & $14.03^{*}$ & & \\
\hline $2 \mathrm{H}-6$ & 1.01 & 14.03 & 15.04 & 13.96 & 14.96 & Yes & $0^{*}$ & $1.01^{*}$ & & & $14.03^{*}$ & $15.04^{*}$ & & \\
\hline $2 \mathrm{H}-7$ & 0.64 & 15.04 & 15.68 & 14.96 & 15.59 & Yes & $0^{*}$ & $0.64^{*}$ & & & $15.04^{*}$ & $15.68^{*}$ & & \\
\hline $2 \mathrm{H}-\mathrm{CC}$ & 0.11 & 15.68 & 15.79 & 15.59 & 15.7 & Yes & $0^{*}$ & $0.11^{*}$ & & & $15.68^{*}$ & $15.79^{*}$ & & \\
\hline $3 \mathrm{H}-1$ & 1.46 & 15.7 & 17.16 & 15.7 & 17.13 & Yes & $0^{\dagger}$ & $0.66^{\dagger}$ & $0.66^{\dagger}$ & $1.16^{\dagger}$ & $15.7^{\dagger}$ & $16.36^{\dagger}$ & $16.36^{\dagger}$ & $16.86^{\dagger}$ \\
\hline $3 \mathrm{H}-1$ & 1.46 & 15.7 & 17.16 & 15.7 & 17.13 & Yes & $1.35^{*}$ & $1.46^{*}$ & & & $17.05^{*}$ & $17.16^{*}$ & & \\
\hline $3 \mathrm{H}-2$ & 1.23 & 17.16 & 18.39 & 17.13 & 18.34 & Yes & $0^{*}$ & $1.23^{*}$ & & & $17.16^{*}$ & $18.39^{*}$ & & \\
\hline $3 \mathrm{H}-3$ & 0.49 & 18.39 & 18.88 & 18.34 & 18.82 & Yes & $0^{*}$ & $0.49^{*}$ & & & $18.39^{*}$ & $18.88^{*}$ & & \\
\hline $3 \mathrm{H}-\mathrm{CC}$ & 0.29 & 18.88 & 19.17 & 18.82 & 19.1 & Yes & $0^{*}$ & $0.29^{*}$ & & & $18.88^{*}$ & $19.17^{*}$ & & \\
\hline $4 \mathrm{H}-1$ & 1.44 & 19.1 & 20.54 & 19.1 & 20.53 & Yes & $0^{\dagger}$ & $0.33^{\dagger}$ & $1.08^{*}$ & $1.44^{*}$ & $19.1^{\dagger}$ & $19.43^{\dagger}$ & $20.18^{*}$ & $20.54^{x}$ \\
\hline $4 \mathrm{H}-2$ & 1.38 & 20.54 & 21.92 & 20.53 & 21.91 & Yes & $1.12^{*}$ & $1.44^{*}$ & $0^{*}$ & $1.12^{*}$ & $21.66^{*}$ & $21.98^{*}$ & $20.54^{*}$ & $21.66^{*}$ \\
\hline $4 \mathrm{H}-3$ & 1.11 & 21.92 & 23.03 & 21.91 & 23.01 & Yes & $0^{*}$ & $1.11^{*}$ & & & $21.92^{\star}$ & $23.03^{*}$ & & \\
\hline $4 \mathrm{H}-4$ & 0.92 & 23.03 & 23.95 & 23.01 & 23.93 & Yes & $0^{*}$ & $0.92^{*}$ & & & $23.03^{*}$ & $23.95^{*}$ & & \\
\hline $4 \mathrm{H}-5$ & 0.93 & 23.95 & 24.88 & 23.93 & 24.86 & Yes & $0^{*}$ & $0.93^{*}$ & & & $23.95^{*}$ & $24.88^{*}$ & & \\
\hline $4 \mathrm{H}-6$ & 1.5 & 24.88 & 26.38 & 24.86 & 26.35 & Yes & $0^{*}$ & $1.5^{*}$ & & & $24.88^{*}$ & $26.38^{*}$ & & \\
\hline $4 \mathrm{H}-\mathrm{CC}$ & 0.15 & 26.38 & 26.53 & 26.35 & 26.5 & Yes & $0^{*}$ & $0.15^{*}$ & & & $26.38^{*}$ & $26.53^{*}$ & & \\
\hline $5 \mathrm{H}-1$ & 1.26 & 26.5 & 27.76 & 26.5 & 27.76 & Yes & $0^{\dagger}$ & $0.2^{\dagger}$ & & & $26.5^{\dagger}$ & $26.7^{\dagger}$ & & \\
\hline $5 \mathrm{H}-1$ & 1.26 & 26.5 & 27.76 & 26.5 & 27.76 & Yes & $0.25^{*}$ & $1.26^{*}$ & & & $26.75^{*}$ & $27.76^{\star}$ & & \\
\hline $5 \mathrm{H}-2$ & 1.29 & 27.76 & 29.05 & 27.76 & 29.05 & Yes & $0^{*}$ & $1.29^{*}$ & & & $27.76^{\star}$ & $29.05^{*}$ & & \\
\hline $5 \mathrm{H}-3$ & 1.32 & 29.05 & 30.37 & 29.05 & 30.37 & Yes & $0^{*}$ & $1.32^{*}$ & & & $29.05^{*}$ & $30.37^{*}$ & & \\
\hline $5 \mathrm{H}-4$ & 1.27 & 30.37 & 31.64 & 30.37 & 31.64 & Yes & $0^{*}$ & $1.27^{*}$ & & & $30.37^{*}$ & $31.64^{*}$ & & \\
\hline $5 \mathrm{H}-5$ & 1.35 & 31.64 & 32.99 & 31.64 & 32.99 & Yes & $0^{*}$ & $1.35^{*}$ & & & $31.64^{*}$ & $32.99^{*}$ & & \\
\hline $5 \mathrm{H}-6$ & 1.51 & 32.99 & 34.5 & 32.99 & 34.5 & Yes & $0^{*}$ & $1.51^{*}$ & & & $32.99^{*}$ & $34.5^{*}$ & & \\
\hline $5 \mathrm{H}-7$ & 0.53 & 34.5 & 35.03 & 34.5 & 35.03 & Yes & $0^{*}$ & $0.53^{*}$ & & & $34.5^{*}$ & $35.03^{*}$ & & \\
\hline $5 \mathrm{H}-\mathrm{CC}$ & 0.2 & 35.03 & 35.23 & 35.03 & 35.23 & Yes & $0^{*}$ & $0.2^{*}$ & & & $35.03^{*}$ & $35.23^{*}$ & & \\
\hline $6 \mathrm{H}-1$ & 1.05 & 35.4 & 36.45 & 35.4 & 36.44 & Yes & $0^{\dagger}$ & $0.53^{\dagger}$ & & & $35.4^{\dagger}$ & $35.93^{\dagger}$ & & \\
\hline $6 \mathrm{H}-1$ & 1.05 & 35.4 & 36.45 & 35.4 & 36.44 & Yes & $0.58^{*}$ & $1.05^{*}$ & & & $35.98^{*}$ & $36.45^{*}$ & & \\
\hline $6 \mathrm{H}-2$ & 1.29 & 36.45 & 37.74 & 36.44 & 37.73 & Yes & $0^{*}$ & $1.29^{*}$ & & & $36.45^{*}$ & $37.74^{*}$ & & \\
\hline $6 \mathrm{H}-3$ & 1.37 & 37.74 & 39.11 & 37.73 & 39.09 & Yes & $0^{*}$ & $1.37^{*}$ & & & $37.74^{*}$ & $39.11^{*}$ & & \\
\hline $6 \mathrm{H}-4$ & 1.15 & 39.11 & 40.26 & 39.09 & 40.23 & Yes & $0^{*}$ & $1.15^{*}$ & & & $39.11^{*}$ & $40.26^{*}$ & & \\
\hline $6 \mathrm{H}-5$ & 1.51 & 40.26 & 41.77 & 40.23 & 41.74 & Yes & $0^{*}$ & $1.51^{*}$ & & & $40.26^{*}$ & $41.77^{\star}$ & & \\
\hline $6 \mathrm{H}-6$ & 0.67 & 41.77 & 42.44 & 41.74 & 42.4 & Yes & $0^{*}$ & $0.67^{*}$ & & & $41.77^{*}$ & $42.44^{*}$ & & \\
\hline $6 \mathrm{H}-\mathrm{CC}$ & 0.2 & 42.44 & 42.64 & 42.4 & 42.6 & Yes & $0^{*}$ & $0.2^{*}$ & & & $42.44^{*}$ & $42.64^{*}$ & & \\
\hline $7 \mathrm{H}-2$ & 1.5 & 44.1 & 45.6 & 44.08 & 45.57 & Yes & $0.68^{*}$ & $1.5^{*}$ & & & $44.78^{*}$ & $45.6^{*}$ & & \\
\hline $7 \mathrm{H}-3$ & 1.5 & 45.6 & 47.1 & 45.57 & 47.05 & Yes & $0^{*}$ & $1^{*}$ & & & $45.6^{*}$ & $46.6^{\star}$ & & \\
\hline $7 \mathrm{H}-4$ & 1.5 & 47.1 & 48.6 & 47.05 & 48.54 & Yes & $0^{*}$ & $5^{*}$ & & & $47.1^{*}$ & $52.1^{*}$ & & \\
\hline $7 \mathrm{H}-5$ & 0.62 & 48.6 & 49.22 & 48.54 & 49.15 & Yes & $0^{*}$ & $0.62^{*}$ & & & $48.6^{*}$ & $49.22^{*}$ & & \\
\hline $7 \mathrm{H}-\mathrm{CC}$ & 0.15 & 49.22 & 49.37 & 49.15 & 49.3 & Yes & $0^{*}$ & $0.15^{*}$ & & & $49.22^{*}$ & $49.37^{\star}$ & & \\
\hline $8 \mathrm{H}-1$ & 1.5 & 49.3 & 50.8 & 49.3 & 50.79 & Yes & $0^{\dagger}$ & $0.45^{\dagger}$ & & & $49.3^{\dagger}$ & $49.75^{\dagger}$ & & \\
\hline $8 \mathrm{H}-1$ & 1.5 & 49.3 & 50.8 & 49.3 & 50.79 & Yes & $0.72^{*}$ & $1.5^{*}$ & & & $50.02^{\star}$ & $50.8^{\star}$ & & \\
\hline $8 \mathrm{H}-2$ & 1.5 & 50.8 & 52.3 & 50.79 & 52.28 & Yes & $0^{*}$ & $1.5^{*}$ & & & $50.8^{*}$ & $52.3^{*}$ & & \\
\hline $8 \mathrm{H}-3$ & 1.28 & 52.3 & 53.58 & 52.28 & 53.56 & Yes & $0^{*}$ & $1.28^{*}$ & & & $52.3^{*}$ & $53.58^{*}$ & & \\
\hline $8 \mathrm{H}-4$ & 1.23 & 53.58 & 54.81 & 53.56 & 54.78 & Yes & $0^{*}$ & $1.23^{*}$ & & & $53.58^{*}$ & $54.81^{*}$ & & \\
\hline $8 \mathrm{H}-5$ & 1.38 & 54.81 & 56.19 & 54.78 & 56.15 & Yes & $0^{*}$ & $1.38^{*}$ & & & $54.81^{*}$ & $56.19^{*}$ & & \\
\hline $8 \mathrm{H}-6$ & 1.11 & 56.19 & 57.3 & 56.15 & 57.26 & Yes & $0^{*}$ & $1.11^{*}$ & & & $56.19^{\star}$ & $57.3^{*}$ & & \\
\hline $8 \mathrm{H}-7$ & 0.67 & 57.3 & 57.97 & 57.26 & 57.92 & Yes & $0^{*}$ & $0.67^{*}$ & & & $57.3^{*}$ & $57.97^{*}$ & & \\
\hline $8 \mathrm{H}-\mathrm{CC}$ & 0.18 & 57.97 & 58.15 & 57.92 & 58.1 & Yes & $0^{*}$ & $0.18^{*}$ & & & $57.97^{*}$ & $58.15^{*}$ & & \\
\hline $9 \mathrm{H}-1$ & 1.5 & 58.1 & 59.6 & 58.1 & 59.6 & No & $0^{\dagger}$ & $0.23^{\dagger}$ & & & $58.1^{\dagger}$ & $58.33^{\dagger}$ & & \\
\hline $10 \mathrm{H}-2$ & 1.07 & 69 & 70.07 & 68.99 & 70.05 & Yes & $0.22^{*}$ & $1.07^{*}$ & & & $69.22^{*}$ & $70.07^{*}$ & & \\
\hline $10 \mathrm{H}-3$ & 0.99 & 70.07 & 71.06 & 70.05 & 71.03 & Yes & $0^{*}$ & $0.99^{*}$ & & & $70.07^{*}$ & $71.06^{*}$ & & \\
\hline
\end{tabular}


Table T1 (continued). (Continued on next page.)

\begin{tabular}{|c|c|c|c|c|c|c|c|c|c|c|c|c|c|c|}
\hline \multirow{2}{*}{$\begin{array}{l}\text { Core, } \\
\text { section }\end{array}$} & \multirow{2}{*}{$\begin{array}{l}\text { Recovered } \\
\text { length } \\
(\mathrm{m})\end{array}$} & \multicolumn{2}{|c|}{ Depth CSF-A (m) } & \multicolumn{2}{|c|}{ Depth CSF-B (m) } & \multirow{2}{*}{$\begin{array}{l}\text { Partial } \\
\text { stroke }\end{array}$} & \multicolumn{2}{|c|}{$\begin{array}{c}\text { Fall-in* or flow-in } \\
\text { depth in section }(\mathrm{m}) \\
\end{array}$} & \multicolumn{2}{|c|}{$\begin{array}{l}\text { Possible fall-in* or } \\
\text { flow-in }{ }^{\dagger} \text { depth } \\
\text { in section }(\mathrm{m}) \\
\end{array}$} & $\begin{array}{r}\text { Fall-in* or } \\
\text { depth } \\
\text { CSF-A }\end{array}$ & $\begin{array}{l}\text { flow-in } \\
\text { n hole } \\
(\mathrm{m})\end{array}$ & $\begin{array}{r}\text { Possible f } \\
\text { flow-in } \\
\text { in hole C } \\
\end{array}$ & $\begin{array}{l}\text { all-in* or } \\
\text { depth } \\
\text { SF-A (m) }\end{array}$ \\
\hline & & Top & Bottom & Top & Bottom & & Top & Bottom & Top & Bottom & Top & Bottom & Top & Bottom \\
\hline $10 \mathrm{H}-4$ & 0.85 & 71.06 & 71.91 & 71.03 & 71.88 & Yes & $0^{*}$ & $0.85^{*}$ & & & $71.06^{*}$ & $71.91^{*}$ & & \\
\hline $10 \mathrm{H}-5$ & 1.5 & 71.91 & 73.41 & 71.88 & 73.36 & Yes & $0^{*}$ & $1.5^{*}$ & & & $71.91^{*}$ & $73.41^{*}$ & & \\
\hline $10 \mathrm{H}-6$ & 0.28 & 73.41 & 73.69 & 73.36 & 73.64 & Yes & $0^{*}$ & $0.28^{*}$ & & & $73.41^{*}$ & $73.69^{*}$ & & \\
\hline $10 \mathrm{H}-\mathrm{CC}$ & 0.16 & 73.69 & 73.85 & 73.64 & 73.8 & Yes & $0^{*}$ & $0.16^{*}$ & & & $73.69^{*}$ & $73.85^{*}$ & & \\
\hline $11 \mathrm{H}-3$ & 1.5 & 76.8 & 78.3 & 76.8 & 78.3 & Yes & $0.84^{*}$ & $1.5^{*}$ & & & $77.64^{*}$ & $78.3^{*}$ & & \\
\hline $11 \mathrm{H}-4$ & 1.5 & 78.3 & 79.8 & 78.3 & 79.8 & Yes & $0^{*}$ & $1.5^{*}$ & & & $78.3^{*}$ & $79.8^{*}$ & & \\
\hline $11 \mathrm{H}-5$ & 1.36 & 79.8 & 81.16 & 79.8 & 81.16 & Yes & $0^{*}$ & $1.36^{*}$ & & & $79.8^{*}$ & $81.16^{*}$ & & \\
\hline $11 \mathrm{H}-6$ & 1.05 & 81.16 & 82.21 & 81.16 & 82.21 & Yes & $0^{*}$ & $1.05^{*}$ & & & $81.16^{*}$ & $82.21^{*}$ & & \\
\hline $11 \mathrm{H}-7$ & 0.49 & 82.21 & 82.7 & 82.21 & 82.7 & Yes & $0^{*}$ & $0.49^{*}$ & & & $82.21^{*}$ & $82.7^{*}$ & & \\
\hline $11 \mathrm{H}-\mathrm{CC}$ & 0.2 & 82.7 & 82.9 & 82.7 & 82.9 & Yes & $0^{*}$ & $0.2^{*}$ & & & $82.7^{*}$ & $82.9^{*}$ & & \\
\hline $12 \mathrm{H}-1$ & 1.5 & 82.9 & 84.4 & 82.9 & 84.4 & Yes & & & $0^{\dagger}$ & $0.03^{\dagger}$ & & & $82.9^{\dagger}$ & $82.93^{\dagger}$ \\
\hline $12 \mathrm{H}-4$ & 1.5 & 87.4 & 88.9 & 87.38 & 88.88 & Yes & $0.47^{*}$ & $1.5^{*}$ & & & $87.87^{*}$ & $88.9^{*}$ & & \\
\hline $12 \mathrm{H}-5$ & 1.17 & 88.9 & 90.07 & 88.88 & 90.05 & Yes & $0^{*}$ & $1.17^{*}$ & & & $88.9^{*}$ & $90.07^{*}$ & & \\
\hline $12 \mathrm{H}-6$ & 1.5 & 90.07 & 91.57 & 90.05 & 91.54 & Yes & $0^{*}$ & $1.5^{*}$ & & & $90.07^{*}$ & $91.57^{*}$ & & \\
\hline $12 \mathrm{H}-\mathrm{CC}$ & 0.16 & 91.57 & 91.73 & 91.54 & 91.7 & Yes & $0^{*}$ & $0.16^{*}$ & & & $91.57^{*}$ & $91.73^{*}$ & & \\
\hline $13 \mathrm{H}-1$ & 1.26 & 91.7 & 92.96 & 91.7 & 92.94 & Yes & $0^{\dagger}$ & $0.28^{\dagger}$ & & & $91.7^{\dagger}$ & $91.98^{\dagger}$ & & \\
\hline $13 \mathrm{H}-2$ & 1.41 & 92.96 & 94.37 & 92.94 & 94.32 & Yes & $0^{*}$ & $1.41^{*}$ & & & $92.96^{*}$ & $94.37^{*}$ & & \\
\hline $13 \mathrm{H}-3$ & 1.51 & 94.37 & 95.88 & 94.32 & 95.8 & Yes & $0^{*}$ & $1.51^{*}$ & & & $94.37^{*}$ & $95.88^{*}$ & & \\
\hline $13 \mathrm{H}-\mathrm{CC}$ & 0.1 & 95.88 & 95.98 & 95.8 & 95.9 & Yes & $0^{*}$ & $0.1^{*}$ & & & $95.88^{*}$ & $95.98^{*}$ & & \\
\hline $14 \mathrm{H}-1$ & 1.5 & 95.9 & 97.4 & 95.9 & 97.34 & No & $0^{\dagger}$ & $0.1^{\dagger}$ & $0.1^{\dagger}$ & $1.14^{\dagger}$ & $95.9^{\dagger}$ & $96^{\dagger}$ & $96^{\dagger}$ & $97.04^{\dagger}$ \\
\hline $16 \mathrm{H}-4$ & 1.5 & 119.4 & 120.9 & 119.34 & 120.83 & Yes & $1.15^{*}$ & $1.5^{*}$ & $0.76^{*}$ & $1.15^{*}$ & $120.55^{*}$ & $120.9^{*}$ & $120.16^{*}$ & $120.55^{\star}$ \\
\hline $16 \mathrm{H}-5$ & 1.22 & 120.9 & 122.12 & 120.83 & 122.03 & Yes & $0^{*}$ & $1.22^{*}$ & & & $120.9^{*}$ & $122.12^{*}$ & & \\
\hline $16 \mathrm{H}-\mathrm{CC}$ & 0.17 & 122.12 & 122.29 & 122.03 & 122.2 & Yes & $0^{*}$ & $0.17^{*}$ & & & $122.12^{*}$ & $122.29^{*}$ & & \\
\hline $17 \mathrm{H}-1$ & 1.5 & 122.2 & 123.7 & 122.2 & 123.69 & Yes & $0.09^{*}$ & $1.5^{*}$ & & & $122.29^{*}$ & $123.7^{*}$ & & \\
\hline $17 \mathrm{H}-2$ & 0.94 & 123.7 & 124.64 & 123.69 & 124.63 & Yes & $0^{*}$ & $0.94^{*}$ & & & $123.7^{*}$ & $124.64^{*}$ & & \\
\hline $17 \mathrm{H}-3$ & 0.25 & 124.64 & 124.89 & 124.63 & 124.88 & Yes & $0^{*}$ & $0.25^{*}$ & & & $124.64^{*}$ & 124.89* & & \\
\hline $17 \mathrm{H}-4$ & 1.5 & 124.89 & 126.39 & 124.88 & 126.37 & Yes & $0^{*}$ & $1.5^{*}$ & & & $124.89^{*}$ & $126.39^{*}$ & & \\
\hline $17 \mathrm{H}-\mathrm{CC}$ & 0.13 & 126.39 & 126.52 & 126.37 & 126.5 & Yes & $0^{*}$ & $0.13^{*}$ & & & $126.39^{*}$ & $126.52^{*}$ & & \\
\hline $18 \mathrm{H}-4$ & 1.5 & 131 & 132.5 & 130.89 & 132.35 & No & & & $0.2^{*}$ & $1.45^{*}$ & & & $131.2^{*}$ & $132.45^{\star}$ \\
\hline $19 \mathrm{H}-1$ & 1.5 & 136 & 137.5 & 136 & 137.5 & No & & & $0^{\dagger}$ & $0.05^{\dagger}$ & & & $136^{\dagger}$ & $136.05^{\dagger}$ \\
\hline $19 \mathrm{H}-4$ & 1.46 & 140.5 & 141.96 & 140.5 & 141.96 & No & & & $0.98^{*}$ & $1.46^{*}$ & & & $141.48^{*}$ & $141.96^{*}$ \\
\hline $19 \mathrm{H}-5$ & 1.45 & 141.96 & 143.41 & 141.96 & 143.41 & No & $0.97^{*}$ & $1.45^{*}$ & $0^{*}$ & $0.97^{*}$ & $142.93^{*}$ & $143.41^{*}$ & $141.96^{*}$ & $142.93^{\prime}$ \\
\hline $19 \mathrm{H}-6$ & 1.49 & 143.41 & 144.9 & 143.41 & 144.9 & No & $0^{*}$ & $1.49^{*}$ & & & $143.41^{*}$ & $144.9^{*}$ & & \\
\hline $19 \mathrm{H}-\mathrm{CC}$ & 0.12 & 144.9 & 145.02 & 144.9 & 145.02 & No & $0^{*}$ & $0.12^{*}$ & & & $144.9^{*}$ & $145.02^{*}$ & & \\
\hline $20 \mathrm{H}-\mathrm{CC}$ & 0.28 & 154.85 & 155.13 & 154.72 & 155 & No & $0^{*}$ & $0.28^{*}$ & & & $154.85^{*}$ & $155.13^{*}$ & & \\
\hline $21 \mathrm{H}-1$ & 1.45 & 155 & 156.45 & 155 & 156.45 & Yes & $0.22^{*}$ & $1.45^{*}$ & & & $155.22^{*}$ & $156.45^{*}$ & & \\
\hline $21 \mathrm{H}-2$ & 1.45 & 156.45 & 157.9 & 156.45 & 157.89 & Yes & $0^{*}$ & $1.45^{*}$ & & & $156.45^{*}$ & $157.9^{*}$ & & \\
\hline $21 \mathrm{H}-3$ & 0.68 & 157.9 & 158.58 & 157.89 & 158.57 & Yes & $0^{*}$ & $0.68^{*}$ & & & $157.9^{*}$ & $158.58^{*}$ & & \\
\hline $21 \mathrm{H}-\mathrm{CC}$ & 0.13 & 158.58 & 158.71 & 158.57 & 158.7 & Yes & $0^{*}$ & $0.13^{*}$ & & & $158.58^{*}$ & $158.71^{*}$ & & \\
\hline $22 \mathrm{H}-1$ & 1.52 & 158.7 & 160.22 & 158.7 & 160.21 & Yes & $0^{\dagger}$ & $0.27^{\dagger}$ & & & $158.7^{\dagger}$ & $158.97^{\dagger}$ & & \\
\hline $23 \mathrm{H}-\mathrm{CC}$ & 0.08 & 166.82 & 166.9 & 166.82 & 166.9 & Yes & $0^{*}$ & $0.08^{*}$ & & & $166.82^{*}$ & $166.9^{*}$ & & \\
\hline $24 \mathrm{H}-1$ & 0.95 & 166.9 & 167.85 & 166.9 & 167.83 & Yes & $0.06^{*}$ & $0.95^{*}$ & & & $166.96^{*}$ & $167.85^{*}$ & & \\
\hline $24 \mathrm{H}-2$ & 0.76 & 167.85 & 168.61 & 167.83 & 168.58 & Yes & $0^{*}$ & $0.76^{*}$ & & & $167.85^{*}$ & $168.61^{*}$ & & \\
\hline $24 \mathrm{H}-3$ & 0.99 & 168.61 & 169.6 & 168.58 & 169.55 & Yes & $0^{*}$ & $0.99^{*}$ & & & $168.61^{*}$ & $169.6^{*}$ & & \\
\hline $24 \mathrm{H}-4$ & 1.01 & 169.6 & 170.61 & 169.55 & 170.54 & Yes & $0^{*}$ & $1.01^{*}$ & & & $169.6^{*}$ & $170.61^{*}$ & & \\
\hline $24 \mathrm{H}-\mathrm{CC}$ & 0.16 & 170.61 & 170.77 & 170.54 & 170.7 & Yes & $0^{*}$ & $0.16^{*}$ & & & $170.61^{*}$ & $170.77^{*}$ & & \\
\hline $340-U 139$ & & & & & & & & & & & & & & \\
\hline $3 \mathrm{H}-1$ & 1.5 & 14.6 & 16.1 & 14.6 & 16.07 & No & $0^{\dagger}$ & $0.3^{\dagger}$ & $0.3^{\dagger}$ & $1.5^{\dagger}$ & $14.6^{\dagger}$ & $14.9^{\dagger}$ & $14.9^{\dagger}$ & $16.1^{\dagger}$ \\
\hline $3 \mathrm{H}-2$ & 1.5 & 16.1 & 17.6 & 16.07 & 17.53 & No & & & $0^{\dagger}$ & $0.97^{\dagger}$ & & & $16.1^{\dagger}$ & $17.07^{\dagger}$ \\
\hline $7 \mathrm{H}-7$ & 0.48 & 61.6 & 62.08 & 61.49 & 61.96 & No & $0.04^{*}$ & $0.48^{*}$ & & & $61.64^{*}$ & $62.08^{*}$ & & \\
\hline 7H-CC & 0.14 & 62.08 & 62.22 & 61.96 & 62.1 & No & $0^{*}$ & $0.14^{*}$ & & & $62.08^{*}$ & $62.22^{*}$ & & \\
\hline $8 \mathrm{H}-1$ & 1.3 & 62.1 & 63.4 & 62.1 & 63.39 & Yes & $1.1^{*}$ & $1.3^{*}$ & & & $63.2^{*}$ & $63.4^{*}$ & & \\
\hline $8 \mathrm{H}-2$ & 1.23 & 63.4 & 64.63 & 63.39 & 64.61 & Yes & $0^{*}$ & $1.23^{*}$ & & & $63.4^{*}$ & $64.63^{*}$ & & \\
\hline $8 \mathrm{H}-3$ & 1.34 & 64.63 & 65.97 & 64.61 & 65.94 & Yes & $0^{*}$ & $0.34^{*}$ & & & $64.63^{*}$ & $64.97^{*}$ & & \\
\hline $8 \mathrm{H}-4$ & 1.31 & 65.97 & 67.28 & 65.94 & 67.24 & Yes & $0^{*}$ & $1.31^{*}$ & & & $65.97^{*}$ & $67.28^{*}$ & & \\
\hline $8 \mathrm{H}-5$ & 1.2 & 67.28 & 68.48 & 67.24 & 68.44 & Yes & $0^{*}$ & $1.2^{*}$ & & & $67.28^{*}$ & $68.48^{*}$ & & \\
\hline $8 \mathrm{H}-6$ & 1.5 & 68.48 & 69.98 & 68.44 & 69.93 & Yes & $0^{*}$ & $1.5^{*}$ & & & $68.48^{*}$ & $69.98^{*}$ & & \\
\hline $8 \mathrm{H}-7$ & 0.69 & 69.98 & 70.67 & 69.93 & 70.61 & Yes & $0^{*}$ & $0.69^{*}$ & & & $69.98^{*}$ & $70.67^{*}$ & & \\
\hline $8 \mathrm{H}-\mathrm{CC}$ & 0.19 & 70.67 & 70.86 & 70.61 & 70.8 & Yes & $0^{*}$ & $0.19^{*}$ & & & $70.67^{*}$ & $70.86^{*}$ & & \\
\hline $9 \mathrm{H}-1$ & 1.5 & 70.8 & 72.3 & 70.8 & 72.23 & No & $0^{\dagger}$ & $0.12^{\dagger}$ & & & $70.8^{\dagger}$ & $70.92^{\dagger}$ & & \\
\hline $10 \mathrm{H}-7$ & 0.81 & 89.18 & 89.99 & 88.78 & 89.55 & No & & & $0^{*}$ & $0.81^{*}$ & & & $89.18^{*}$ & $89.99^{*}$ \\
\hline $10 \mathrm{H}-\mathrm{CC}$ & 0.26 & 89.99 & 90.25 & 89.55 & 89.8 & No & & & $0^{*}$ & $0.26^{*}$ & & & 89.99* & $90.25^{*}$ \\
\hline $11 \mathrm{H}-1$ & 0.91 & 89.8 & 90.71 & 89.8 & 90.68 & Yes & & & $0^{*}$ & $0.92^{*}$ & & & $89.8^{*}$ & $90.72^{*}$ \\
\hline $11 \mathrm{H}-2$ & 1.19 & 90.71 & 91.9 & 90.68 & 91.84 & Yes & $0^{*}$ & $1.19^{*}$ & & & $90.71^{*}$ & $91.9^{*}$ & & \\
\hline $11 \mathrm{H}-\mathrm{CC}$ & 0.27 & 91.9 & 92.17 & 91.84 & 92.1 & Yes & $0^{*}$ & $0.27^{*}$ & & & $91.9^{*}$ & $92.17^{*}$ & & \\
\hline $12 \mathrm{H}-1$ & 0.33 & 92.1 & 92.43 & 92.1 & 92.43 & Yes & $0^{\dagger}$ & $0.12^{\dagger}$ & & & $92.1^{\dagger}$ & $92.22^{\dagger}$ & & \\
\hline $12 \mathrm{H}-1$ & 0.33 & 92.1 & 92.43 & 92.1 & 92.43 & Yes & $0.12^{*}$ & $0.33^{*}$ & & & $92.22^{*}$ & $92.43^{*}$ & & \\
\hline
\end{tabular}


Table T1 (continued). (Continued on next page.)

\begin{tabular}{|c|c|c|c|c|c|c|c|c|c|c|c|c|c|c|}
\hline \multirow{2}{*}{$\begin{array}{l}\text { Core, } \\
\text { section }\end{array}$} & \multirow{2}{*}{$\begin{array}{l}\text { Recovered } \\
\text { length } \\
\text { (m) }\end{array}$} & \multicolumn{2}{|c|}{ Depth CSF-A (m) } & \multicolumn{2}{|c|}{ Depth CSF-B (m) } & \multirow{2}{*}{$\begin{array}{l}\text { Partial } \\
\text { stroke }\end{array}$} & \multicolumn{2}{|c|}{$\begin{array}{c}\text { Fall-in }^{*} \text { or flow-in } \\
\text { depth in section }(\mathrm{m}) \\
\end{array}$} & \multicolumn{2}{|c|}{$\begin{array}{l}\text { Possible fall-in* or } \\
\text { flow-in }{ }^{\dagger} \text { depth } \\
\text { in section }(\mathrm{m})\end{array}$} & $\begin{array}{l}\text { Fall-in* or } \\
\text { depth } \\
\text { CSF-A }\end{array}$ & $\begin{array}{l}r_{\text {flow-in }}^{\dagger} \\
\text { in hole } \\
\mathrm{A}(\mathrm{m}) \\
\end{array}$ & $\begin{array}{r}\text { Possible f } \\
\text { flow-in' } \\
\text { in hole C } \\
\end{array}$ & $\begin{array}{l}\text { all-in* or } \\
\text { depth } \\
\text { SF-A }(m)\end{array}$ \\
\hline & & Top & Bottom & Top & Bottom & & Top & Bottom & Top & Bottom & Top & Bottom & Top & Bottom \\
\hline $12 \mathrm{H}-2$ & 1.5 & 92.43 & 93.93 & 92.43 & 93.91 & Yes & $0^{*}$ & $1.5^{*}$ & & & $92.43^{*}$ & $93.93^{*}$ & & \\
\hline $12 \mathrm{H}-3$ & 1.04 & 93.93 & 94.97 & 93.91 & 94.93 & Yes & $0^{*}$ & $1.04^{*}$ & & & $93.93^{*}$ & $94.97 *$ & & \\
\hline $12 \mathrm{H}-\mathrm{CC}$ & 0.17 & 94.97 & 95.14 & 94.93 & 95.1 & Yes & $0^{*}$ & $0.17^{*}$ & & & $94.97^{*}$ & $95.14^{*}$ & & \\
\hline $13 \mathrm{H}-2$ & 1.32 & 96.6 & 97.92 & 96.58 & 97.89 & Yes & $0.99^{*}$ & $1.32^{*}$ & & & $97.59^{*}$ & $97.92^{*}$ & & \\
\hline $13 \mathrm{H}-3$ & 1.29 & 97.92 & 99.21 & 97.89 & 99.16 & Yes & $0^{*}$ & $1.29 *$ & & & $97.92^{*}$ & $99.21^{*}$ & & \\
\hline $13 \mathrm{H}-4$ & 1.13 & 99.21 & 100.34 & 99.16 & 100.28 & Yes & $0^{*}$ & $1.13^{*}$ & & & $99.21^{*}$ & $100.34^{*}$ & & \\
\hline $13 \mathrm{H}-5$ & 1.0 & 100.34 & 101.34 & 100.28 & 101.27 & Yes & $0^{*}$ & $1^{*}$ & & & $100.34^{*}$ & $101.34^{*}$ & & \\
\hline $13 \mathrm{H}-6$ & 1.48 & 101.34 & 102.82 & 101.27 & 102.73 & Yes & $0^{*}$ & $1.48^{*}$ & & & $101.34^{*}$ & $102.82^{*}$ & & \\
\hline $13 \mathrm{H}-\mathrm{CC}$ & 0.17 & 102.82 & 102.99 & 102.73 & 102.9 & Yes & $0^{*}$ & $0.17^{*}$ & & & $102.82^{*}$ & $102.99 *$ & & \\
\hline $14 \mathrm{H}-1$ & 1.5 & 102.9 & 104.4 & 102.9 & 104.38 & Yes & $0^{\dagger}$ & $0.19^{\dagger}$ & & & $102.9^{\dagger}$ & $103.09^{\dagger}$ & & \\
\hline $14 \mathrm{H}-1$ & 1.5 & 102.9 & 104.4 & 102.9 & 104.38 & Yes & $1.28^{*}$ & $1.5^{*}$ & & & $104.18^{*}$ & $104.4^{*}$ & & \\
\hline $14 \mathrm{H}-2$ & 1.5 & 104.4 & 105.9 & 104.38 & 105.87 & Yes & $0^{*}$ & $1.5^{*}$ & & & $104.4^{*}$ & $105.9^{*}$ & & \\
\hline $14 \mathrm{H}-3$ & 1.5 & 105.9 & 107.4 & 105.87 & 107.36 & Yes & $0^{*}$ & $1.5^{*}$ & & & $105.9^{*}$ & $107.4^{*}$ & & \\
\hline $14 \mathrm{H}-4$ & 1.5 & 107.4 & 108.9 & 107.36 & 108.84 & Yes & $0^{*}$ & $1.5^{*}$ & & & $107.4^{\star}$ & $108.9^{*}$ & & \\
\hline $14 \mathrm{H}-5$ & 1.5 & 108.9 & 110.4 & 108.84 & 110.33 & Yes & $0^{*}$ & $1.5^{*}$ & & & $108.9^{\star}$ & $110.4^{*}$ & & \\
\hline $14 \mathrm{H}-6$ & 1.53 & 110.4 & 111.93 & 110.33 & 111.84 & Yes & $0^{*}$ & $1.53^{*}$ & & & $110.4^{*}$ & 111.93* & & \\
\hline $14 \mathrm{H}-\mathrm{CC}$ & 0.16 & 111.93 & 112.09 & 111.84 & 112 & Yes & $0^{*}$ & $0.16^{*}$ & & & 111.93* & $112.09^{*}$ & & \\
\hline $15 \mathrm{H}-1$ & 1.5 & 112 & 113.5 & 112 & 113.43 & No & & & $0^{\dagger}$ & $0.03^{\dagger}$ & & & $112^{\dagger}$ & $112.03^{\dagger}$ \\
\hline $15 \mathrm{H}-6$ & 1.52 & 119.52 & 121.04 & 119.17 & 120.62 & No & $0.22^{*}$ & $1.52^{*}$ & & & $119.74^{*}$ & $121.04^{*}$ & & \\
\hline $15 \mathrm{H}-7$ & 0.69 & 121.04 & 121.73 & 120.62 & 121.28 & No & $0^{*}$ & $0.69^{*}$ & & & $121.04^{*}$ & $121.73^{*}$ & & \\
\hline $15 \mathrm{H}-\mathrm{CC}$ & 0.23 & 121.73 & 121.96 & 121.28 & 121.5 & No & $0^{*}$ & $0.23^{*}$ & & & $121.73^{*}$ & $121.96^{*}$ & & \\
\hline $16 \mathrm{H}-1$ & 1.5 & 121.5 & 123 & 121.5 & 122.92 & No & $0^{\dagger}$ & $0.32^{\dagger}$ & & & $121.5^{\dagger}$ & $121.82^{\dagger}$ & & \\
\hline $18 \mathrm{H}-2$ & 1.5 & 142 & 143.5 & 141.94 & 143.39 & Yes & $0.57^{*}$ & $1.5^{*}$ & & & $142.57^{*}$ & $143.5^{*}$ & & \\
\hline $18 \mathrm{H}-3$ & 1.5 & 143.5 & 145 & 143.39 & 144.84 & Yes & $0^{*}$ & $1.5^{*}$ & & & $143.5^{\star}$ & $145^{*}$ & & \\
\hline $18 \mathrm{H}-4$ & 1.5 & 145 & 146.5 & 144.84 & 146.28 & Yes & $0^{*}$ & $1.5^{*}$ & & & $145^{*}$ & $146.5^{*}$ & & \\
\hline $18 \mathrm{H}-5$ & 1.5 & 146.5 & 148 & 146.28 & 147.73 & Yes & $0^{*}$ & $1.5^{*}$ & & & $146.5^{*}$ & $148^{*}$ & & \\
\hline $18 \mathrm{H}-6$ & 1.5 & 148 & 149.5 & 147.73 & 149.17 & Yes & $0^{*}$ & $1.5^{*}$ & & & $148^{*}$ & $149.5^{*}$ & & \\
\hline $18 \mathrm{H}-7$ & 0.7 & 149.5 & 150.2 & 149.17 & 149.85 & Yes & $0^{*}$ & $0.7^{*}$ & & & $149.5^{*}$ & $150.2^{*}$ & & \\
\hline $18 \mathrm{H}-\mathrm{CC}$ & 0.16 & 150.2 & 150.36 & 149.85 & 150 & Yes & $0^{*}$ & $0.16^{*}$ & & & $150.2^{*}$ & $150.36^{*}$ & & \\
\hline $19 \mathrm{H}-1$ & 0.26 & 150 & 150.26 & 150 & 150.26 & Yes & $0^{\dagger}$ & $0.26^{\dagger}$ & & & $150^{\dagger}$ & $150.26^{\dagger}$ & & \\
\hline $19 \mathrm{H}-\mathrm{CC}$ & 0.05 & 150.26 & 150.31 & 150.26 & 150.31 & Yes & $0^{\dagger}$ & $0.05^{\dagger}$ & & & $150.26^{\dagger}$ & $150.31^{\dagger}$ & & \\
\hline $20 \mathrm{H}-1$ & 1.5 & 153.4 & 154.9 & 153.4 & 154.89 & Yes & $0^{\dagger}$ & $0.38^{\dagger}$ & & & $153.4^{\dagger}$ & $153.78^{\dagger}$ & & \\
\hline $21 \mathrm{H}-1$ & 0.79 & 160.6 & 161.39 & 160.6 & 161.38 & Yes & $0^{*}$ & $0.79 *$ & & & $160.6^{*}$ & 161.39* & & \\
\hline $21 \mathrm{H}-2$ & 1.17 & 161.39 & 162.56 & 161.38 & 162.53 & Yes & $0^{*}$ & $1.17^{*}$ & & & 161.39* & $162.56^{*}$ & & \\
\hline $21 \mathrm{H}-3$ & 1.56 & 162.56 & 164.12 & 162.53 & 164.06 & Yes & $0^{*}$ & $1.56^{*}$ & & & $162.56^{*}$ & $164.12^{*}$ & & \\
\hline $21 \mathrm{H}-\mathrm{CC}$ & 0.14 & 164.12 & 164.26 & 164.06 & 164.2 & Yes & $0^{*}$ & $0.14^{*}$ & & & $164.12^{*}$ & $164.26^{*}$ & & \\
\hline $22 \mathrm{H}-1$ & 1.5 & 164.2 & 165.7 & 164.2 & 165.67 & Yes & $0^{*}$ & $1.5^{*}$ & & & $164.2^{*}$ & $165.7^{*}$ & & \\
\hline $22 \mathrm{H}-2$ & 1.49 & 165.7 & 167.19 & 165.67 & 167.12 & Yes & $0^{*}$ & $1.49^{*}$ & & & $165.7^{*}$ & $167.19^{*}$ & & \\
\hline $22 \mathrm{H}-\mathrm{CC}$ & 0.08 & 167.19 & 167.27 & 167.12 & 167.2 & Yes & $0^{*}$ & $0.08^{*}$ & & & 167.19* & $167.27^{*}$ & & \\
\hline $23 \mathrm{H}-1$ & 1.55 & 167.2 & 168.75 & 167.2 & 168.74 & Yes & $0^{\dagger}$ & $0.22^{\dagger}$ & & & $167.2^{\dagger}$ & $167.42^{\dagger}$ & & \\
\hline $23 \mathrm{H}-3$ & 1.47 & 170.22 & 171.69 & 170.19 & 171.65 & Yes & & & $0.62^{*}$ & $1.47^{*}$ & & & $170.84^{*}$ & $171.69^{*}$ \\
\hline $23 \mathrm{H}-4$ & 1.47 & 171.69 & 173.16 & 171.65 & 173.11 & Yes & & & $0^{*}$ & $1.47^{*}$ & & & $171.69^{*}$ & $173.16^{*}$ \\
\hline $23 \mathrm{H}-5$ & 1.46 & 173.16 & 174.62 & 173.11 & 174.56 & Yes & & & $0^{*}$ & $1.46^{*}$ & & & $173.16^{*}$ & $174.62^{*}$ \\
\hline $23 \mathrm{H}-6$ & 0.76 & 174.62 & 175.38 & 174.56 & 175.31 & Yes & $0^{*}$ & $0.76^{*}$ & & & $174.62^{*}$ & $175.38^{*}$ & & \\
\hline $23 \mathrm{H}-\mathrm{CC}$ & 0.09 & 175.38 & 175.47 & 175.31 & 175.4 & Yes & $0^{*}$ & $0.09^{*}$ & & & $175.38^{*}$ & $175.47^{*}$ & & \\
\hline $24 \mathrm{H}-1$ & 1.5 & 175.4 & 176.9 & 175.4 & 176.9 & Yes & $0^{\dagger}$ & $0.03^{\dagger}$ & & & $175.4^{\dagger}$ & $175.43^{\dagger}$ & & \\
\hline $24 \mathrm{H}-3$ & 1.41 & 178.4 & 179.81 & 178.4 & 179.81 & Yes & $0.13^{*}$ & $1.41^{*}$ & & & $178.53^{*}$ & $179.81^{*}$ & & \\
\hline $24 \mathrm{H}-4$ & 1.13 & 179.81 & 180.94 & 179.81 & 180.94 & Yes & $0^{*}$ & $1.13^{*}$ & & & $179.81^{*}$ & $180.94^{*}$ & & \\
\hline $24 \mathrm{H}-5$ & 1.21 & 180.94 & 182.15 & 180.94 & 182.15 & Yes & $0^{*}$ & $1.21^{*}$ & & & $180.94^{*}$ & $182.15^{*}$ & & \\
\hline $24 \mathrm{H}-6$ & 1.5 & 182.15 & 183.65 & 182.15 & 183.65 & Yes & $0^{*}$ & $1.5^{*}$ & & & $182.15^{*}$ & $183.65^{*}$ & & \\
\hline $24 \mathrm{H}-7$ & 0.64 & 183.65 & 184.29 & 183.65 & 184.29 & Yes & $0^{*}$ & $0.64^{*}$ & & & $183.65^{*}$ & $184.29^{*}$ & & \\
\hline $24 \mathrm{H}-\mathrm{CC}$ & 0.21 & 184.29 & 184.5 & 184.29 & 184.5 & Yes & $0^{*}$ & $0.21^{*}$ & & & $184.29^{*}$ & $184.5^{\star}$ & & \\
\hline $25 \mathrm{H}-2$ & 1.5 & 186 & 187.5 & 185.98 & 187.47 & Yes & $0.95^{*}$ & $1.5^{*}$ & & & $186.95^{*}$ & $187.5^{*}$ & & \\
\hline $25 \mathrm{H}-3$ & 0.58 & 187.5 & 188.08 & 187.47 & 188.04 & Yes & $0^{*}$ & $0.58^{*}$ & & & $187.5^{*}$ & $188.08^{*}$ & & \\
\hline $25 \mathrm{H}-4$ & 0.8 & 188.08 & 188.88 & 188.04 & 188.83 & Yes & $0^{*}$ & $0.8^{*}$ & & & $188.08^{*}$ & $188.88^{*}$ & & \\
\hline $25 \mathrm{H}-5$ & 1.14 & 188.88 & 190.02 & 188.83 & 189.96 & Yes & $0^{*}$ & $1.14^{*}$ & & & $188.88^{*}$ & $190.02^{*}$ & & \\
\hline $25 \mathrm{H}-6$ & 1.01 & 190.02 & 191.03 & 189.96 & 190.96 & Yes & $0^{*}$ & $1.01^{*}$ & & & $190.02^{*}$ & $191.03^{*}$ & & \\
\hline $25 \mathrm{H}-7$ & 0.62 & 191.03 & 191.65 & 190.96 & 191.57 & Yes & $0^{*}$ & $0.62^{*}$ & & & 191.03* & $191.65^{*}$ & & \\
\hline $25 \mathrm{H}-\mathrm{CC}$ & 0.13 & 191.65 & 191.78 & 191.57 & 191.7 & Yes & $0^{*}$ & $0.13^{*}$ & & & $191.65^{*}$ & $191.78^{*}$ & & \\
\hline $26 \mathrm{H}-3$ & 1.5 & 194.74 & 196.24 & 194.72 & 196.21 & Yes & $1.42^{*}$ & $1.5^{*}$ & & & $196.16^{*}$ & $196.24^{*}$ & & \\
\hline $26 \mathrm{H}-4$ & 1.16 & 196.24 & 197.4 & 196.21 & 197.36 & Yes & $0^{*}$ & $1.16^{*}$ & & & $196.24^{*}$ & $197.4^{*}$ & & \\
\hline $26 \mathrm{H}-5$ & 0.28 & 197.4 & 197.68 & 197.36 & 197.64 & Yes & $0^{*}$ & $0.28^{*}$ & & & $197.4^{*}$ & $197.68^{*}$ & & \\
\hline $26 \mathrm{H}-\mathrm{CC}$ & 0.06 & 197.68 & 197.74 & 197.64 & 197.7 & Yes & $0^{*}$ & $0.06^{*}$ & & & $197.68^{*}$ & $197.74^{*}$ & & \\
\hline $27 \mathrm{H}-4$ & 1.5 & 202.24 & 203.74 & 202.24 & 203.74 & Yes & $1.3^{*}$ & $1.5^{*}$ & & & $203.54^{*}$ & 203.74* & & \\
\hline $27 \mathrm{H}-\mathrm{CC}$ & 0.16 & 203.74 & 203.9 & 203.74 & 203.9 & Yes & $0^{*}$ & $0.16^{*}$ & & & $203.74^{*}$ & $203.9^{*}$ & & \\
\hline $29 \mathrm{H}-1$ & 1.22 & 205.5 & 206.72 & 205.5 & 206.7 & Yes & $0.93^{*}$ & $1.22^{*}$ & & & 206.43* & $206.72^{*}$ & & \\
\hline $29 \mathrm{H}-2$ & 0.66 & 206.72 & 207.38 & 206.7 & 207.34 & Yes & $0^{*}$ & $0.66^{*}$ & & & $206.72^{*}$ & $207.38^{*}$ & & \\
\hline $29 \mathrm{H}-\mathrm{CC}$ & 0.16 & 207.38 & 207.54 & 207.34 & 207.5 & Yes & $0^{*}$ & $0.16^{*}$ & & & $207.38^{*}$ & $207.54^{*}$ & & \\
\hline
\end{tabular}


Table T1 (continued). (Continued on next page.)

\begin{tabular}{|c|c|c|c|c|c|c|c|c|c|c|c|c|c|c|}
\hline \multirow{2}{*}{$\begin{array}{l}\text { Core, } \\
\text { section }\end{array}$} & \multirow{2}{*}{$\begin{array}{l}\text { Recovered } \\
\text { length } \\
\text { (m) }\end{array}$} & \multicolumn{2}{|c|}{ Depth CSF-A (m) } & \multicolumn{2}{|c|}{ Depth CSF-B (m) } & \multirow{2}{*}{$\begin{array}{l}\text { Partial } \\
\text { stroke }\end{array}$} & \multicolumn{2}{|c|}{$\begin{array}{c}\text { Fall-in }^{*} \text { or flow-in } \\
\text { depth in section }(\mathrm{m}) \\
\end{array}$} & \multicolumn{2}{|c|}{$\begin{array}{l}\text { Possible fall-in* or } \\
\text { flow-in }{ }^{\dagger} \text { depth } \\
\text { in section }(m) \\
\end{array}$} & $\begin{array}{r}\text { Fall-in* or } \\
\text { depth i } \\
\text { CSF-A }\end{array}$ & $\begin{array}{l}\text { flow-in } \\
\text { n hole } \\
(\mathrm{m})\end{array}$ & $\begin{array}{r}\text { Possible } \mathrm{f}^{+} \\
\text {flow-in }^{\dagger} \\
\text { in hole CS }\end{array}$ & $\begin{array}{l}\text { all-in* or } \\
\text { depth } \\
\text { SF-A }(m)\end{array}$ \\
\hline & & Top & Bottom & Top & Bottom & & Top & Bottom & Top & Bottom & Top & Bottom & Top & Bottom \\
\hline 340-U1399 & & & & & & & & & & & & & & \\
\hline $1 \mathrm{H}-4$ & 1.12 & 4.5 & 5.62 & 4.44 & 5.54 & No & $1.08^{*}$ & $1.12^{*}$ & & & $5.58^{*}$ & $5.62^{*}$ & & \\
\hline $1 \mathrm{H}-\mathrm{CC}$ & 0.16 & 5.62 & 5.78 & 5.54 & 5.7 & No & $0^{*}$ & $0.16^{*}$ & & & $5.62^{\star}$ & $5.78^{*}$ & & \\
\hline $2 \mathrm{H}-5$ & 1.5 & 11.7 & 13.2 & 11.49 & 12.93 & No & & & $1.15^{*}$ & $1.5^{*}$ & & & $12.85^{*}$ & $13.2^{*}$ \\
\hline $2 \mathrm{H}-6$ & 1.5 & 13.2 & 14.7 & 12.93 & 14.38 & No & & & $0^{*}$ & $1.5^{*}$ & & & $13.2^{*}$ & $14.7^{*}$ \\
\hline $2 \mathrm{H}-7$ & 0.66 & 14.7 & 15.36 & 14.38 & 15.02 & No & & & $0^{*}$ & $0.66^{*}$ & & & $14.7^{*}$ & $15.36^{*}$ \\
\hline $2 \mathrm{H}-\mathrm{CC}$ & 0.19 & 15.36 & 15.55 & 15.02 & 15.2 & No & & & $0^{*}$ & $0.19^{*}$ & & & $15.36^{*}$ & $15.55^{*}$ \\
\hline $7 \mathrm{H}-6$ & 0.71 & 60.45 & 61.16 & 60.45 & 61.16 & No & $0^{*}$ & $0.71^{*}$ & & & $60.45^{*}$ & $61.16^{*}$ & & \\
\hline $7 \mathrm{H}-\mathrm{CC}$ & 0.2 & 61.16 & 61.36 & 61.16 & 61.36 & No & $0^{*}$ & $0.2^{*}$ & & & $61.16^{*}$ & $61.36^{*}$ & & \\
\hline $8 \mathrm{H}-1$ & 1.5 & 62.7 & 64.2 & 62.7 & 64.19 & Yes & $0^{\dagger}$ & $0.04^{\dagger}$ & & & $62.7^{\dagger}$ & $62.74^{\dagger}$ & & \\
\hline $8 \mathrm{H}-4$ & 1.5 & 67.15 & 68.65 & 67.12 & 68.61 & Yes & $1.3^{*}$ & $1.5^{*}$ & & & $68.45^{*}$ & $68.65^{*}$ & & \\
\hline $8 \mathrm{H}-5$ & 1.5 & 68.65 & 70.15 & 68.61 & 70.1 & Yes & $0^{*}$ & $1.5^{*}$ & & & $68.65^{*}$ & $70.15^{*}$ & & \\
\hline $8 \mathrm{H}-6$ & 1.01 & 70.15 & 71.16 & 70.1 & 71.1 & Yes & $0^{*}$ & $1.01^{*}$ & & & $70.15^{*}$ & $71.16^{*}$ & & \\
\hline $8 \mathrm{H}-7$ & 0.63 & 71.16 & 71.79 & 71.1 & 71.73 & Yes & $0^{*}$ & $0.63^{*}$ & & & $71.16^{*}$ & $71.79^{*}$ & & \\
\hline $8 \mathrm{H}-\mathrm{CC}$ & 0.17 & 71.79 & 71.96 & 71.73 & 71.9 & Yes & $0^{*}$ & $0.17^{*}$ & & & $71.79^{*}$ & $71.96^{*}$ & & \\
\hline $9 \mathrm{H}-6$ & 1.5 & 78.92 & 80.42 & 78.9 & 80.4 & Yes & $1.25^{*}$ & $1.5^{*}$ & & & $80.17^{*}$ & $80.42^{*}$ & & \\
\hline $9 \mathrm{H}-7$ & 0.64 & 80.42 & 81.06 & 80.4 & 81.04 & Yes & $0^{*}$ & $0.64^{*}$ & & & $80.42^{*}$ & $81.06^{*}$ & & \\
\hline $9 \mathrm{H}-\mathrm{CC}$ & 0.16 & 81.06 & 81.22 & 81.04 & 81.2 & Yes & $0^{*}$ & $0.16^{*}$ & & & $81.06^{*}$ & $81.22^{*}$ & & \\
\hline $10 \mathrm{H}-1$ & 1.5 & 81.2 & 82.7 & 81.2 & 82.69 & Yes & $0.87^{*}$ & $1.5^{*}$ & & & $82.07^{*}$ & $82.7^{*}$ & & \\
\hline $10 \mathrm{H}-2$ & 1.41 & 82.7 & 84.11 & 82.69 & 84.09 & Yes & $0^{*}$ & $1.41^{*}$ & & & $82.7^{\star}$ & $84.11^{*}$ & & \\
\hline $10 \mathrm{H}-3$ & 1.29 & 84.11 & 85.4 & 84.09 & 85.37 & Yes & $0^{*}$ & $1.29^{*}$ & & & $84.11^{*}$ & $85.4^{*}$ & & \\
\hline $10 \mathrm{H}-4$ & 1.25 & 85.4 & 86.65 & 85.37 & 86.61 & Yes & $0^{*}$ & $1.25^{*}$ & & & $85.4^{*}$ & $86.65^{*}$ & & \\
\hline $10 \mathrm{H}-5$ & 1.5 & 86.65 & 88.15 & 86.61 & 88.09 & Yes & $0^{*}$ & $1.5^{*}$ & & & $86.65^{*}$ & $88.15^{*}$ & & \\
\hline $10 \mathrm{H}-6$ & 1.52 & 88.15 & 89.67 & 88.09 & 89.6 & Yes & $0^{*}$ & $1.52^{*}$ & & & $88.15^{*}$ & $89.67^{*}$ & & \\
\hline $10 \mathrm{H}-\mathrm{CC}$ & 0.2 & 89.67 & 89.87 & 89.6 & 89.8 & Yes & $0^{*}$ & $0.2^{*}$ & & & $89.67^{*}$ & $89.87^{*}$ & & \\
\hline $11 \mathrm{H}-3$ & 1.52 & 92.7 & 94.22 & 92.65 & 94.14 & Yes & $0^{*}$ & $1.52^{*}$ & & & $92.7^{*}$ & $94.22^{*}$ & & \\
\hline $11 \mathrm{H}-\mathrm{CC}$ & 0.16 & 94.22 & 94.38 & 94.14 & 94.3 & Yes & $0^{*}$ & $0.16^{*}$ & & & $94.22^{*}$ & $94.38^{*}$ & & \\
\hline $12 \mathrm{H}-1$ & 1.52 & 94.3 & 95.82 & 94.3 & 95.81 & Yes & $0^{\dagger}$ & $1.42^{\dagger}$ & & & $94.3^{\dagger}$ & $95.72^{\dagger}$ & & \\
\hline $12 \mathrm{H}-2$ & 1.43 & 95.82 & 97.25 & 95.81 & 97.24 & Yes & $0.77^{*}$ & $1.43^{*}$ & & & $96.59^{*}$ & $97.25^{*}$ & & \\
\hline $12 \mathrm{H}-3$ & 1.4 & 97.25 & 98.65 & 97.24 & 98.63 & Yes & $0^{*}$ & $1.4^{*}$ & & & $97.25^{*}$ & $98.65^{*}$ & & \\
\hline $12 \mathrm{H}-4$ & 1.42 & 98.65 & 100.07 & 98.63 & 100.04 & Yes & $0^{*}$ & $1.42^{*}$ & & & $98.65^{*}$ & $100.07^{*}$ & & \\
\hline $12 \mathrm{H}-5$ & 1.5 & 100.07 & 101.57 & 100.04 & 101.54 & Yes & $0^{*}$ & $1.5^{\star}$ & & & $100.07^{\star}$ & $101.57^{*}$ & & \\
\hline $12 \mathrm{H}-6$ & 1 & 101.57 & 102.57 & 101.54 & 102.53 & Yes & $0^{*}$ & $1^{*}$ & & & $101.57^{*}$ & $102.57^{*}$ & & \\
\hline $12 \mathrm{H}-\mathrm{CC}$ & 0.17 & 102.57 & 102.74 & 102.53 & 102.7 & Yes & $0^{*}$ & $0.17^{*}$ & & & $102.57^{*}$ & $102.74^{*}$ & & \\
\hline $13 \mathrm{H}-3$ & 1.5 & 105.7 & 107.2 & 105.6 & 107.04 & No & & & $0.7^{*}$ & $1.5^{*}$ & & & $106.4^{*}$ & $107.2^{*}$ \\
\hline $13 \mathrm{H}-4$ & 1.53 & 107.2 & 108.73 & 107.04 & 108.52 & No & & & $0^{*}$ & $1.53^{*}$ & & & $107.2^{*}$ & $108.73^{*}$ \\
\hline $13 \mathrm{H}-5$ & 1.46 & 108.73 & 110.19 & 108.52 & 109.93 & No & & & $0^{*}$ & $1.46^{*}$ & & & $108.73^{*}$ & $110.19^{*}$ \\
\hline $13 \mathrm{H}-6$ & 1.53 & 110.19 & 111.72 & 109.93 & 111.41 & No & & & $0^{*}$ & $1.53^{*}$ & & & $110.19^{*}$ & $111.72^{*}$ \\
\hline $13 \mathrm{H}-7$ & 0.54 & 111.72 & 112.26 & 111.41 & 111.93 & No & & & $0^{*}$ & $0.54^{*}$ & & & $111.72^{*}$ & $112.26^{*}$ \\
\hline $13 \mathrm{H}-\mathrm{CC}$ & 0.28 & 112.26 & 112.54 & 111.93 & 112.2 & No & $0^{*}$ & $0.28^{*}$ & & & $112.26^{*}$ & $112.54^{*}$ & & \\
\hline $14 \mathrm{H}-1$ & 0.26 & 112.2 & 112.46 & 112.2 & 112.46 & Yes & $0^{*}$ & $0.26^{*}$ & & & $112.2^{*}$ & $112.46^{*}$ & & \\
\hline $14 \mathrm{H}-2$ & 0.84 & 112.46 & 113.3 & 112.46 & 113.3 & Yes & $0^{*}$ & $0.84^{*}$ & & & $112.46^{*}$ & $113.3^{*}$ & & \\
\hline $14 \mathrm{H}-3$ & 0.43 & 113.3 & 113.73 & 113.3 & 113.73 & Yes & $0^{*}$ & $0.43^{*}$ & & & $113.3^{*}$ & $113.73^{*}$ & & \\
\hline $14 \mathrm{H}-4$ & 0.76 & 113.73 & 114.49 & 113.73 & 114.49 & Yes & $0^{*}$ & $0.76^{*}$ & & & $113.73^{*}$ & $114.49^{*}$ & & \\
\hline $14 \mathrm{H}-\mathrm{CC}$ & 0.21 & 114.49 & 114.7 & 114.49 & 114.7 & Yes & $0^{*}$ & $0.21^{*}$ & & & $114.49^{*}$ & $114.7^{*}$ & & \\
\hline $18 \mathrm{H}-1$ & 1.5 & 128.9 & 130.4 & 128.9 & 130.39 & Yes & $0^{\dagger}$ & $0.22^{\dagger}$ & & & $128.9^{\dagger}$ & $129.12^{\dagger}$ & & \\
\hline $21 \mathrm{H}-3$ & 1.05 & 148.36 & 149.41 & 148.35 & 149.39 & Yes & & & $0.88^{*}$ & $1.05^{*}$ & & & $149.24^{*}$ & $149.41^{*}$ \\
\hline $21 \mathrm{H}-\mathrm{CC}$ & 0.11 & 149.41 & 149.52 & 149.39 & 149.5 & Yes & & & $0^{*}$ & $0.11^{*}$ & & & $149.41^{*}$ & $149.52^{*}$ \\
\hline $22 \mathrm{H}-3$ & 0.97 & 152.27 & 153.24 & 152.25 & 153.21 & Yes & $0^{*}$ & $0.97^{*}$ & & & $152.27^{*}$ & $153.24^{*}$ & & \\
\hline $22 \mathrm{H}-4$ & 1.22 & 153.24 & 154.46 & 153.21 & 154.42 & Yes & $0^{*}$ & $1.22^{*}$ & & & $153.24^{*}$ & $154.46^{*}$ & & \\
\hline $22 \mathrm{H}-5$ & 1.24 & 154.46 & 155.7 & 154.42 & 155.65 & Yes & $0^{*}$ & $1.24^{*}$ & & & $154.46^{*}$ & $155.7^{*}$ & & \\
\hline $22 \mathrm{H}-6$ & 1.4 & 155.7 & 157.1 & 155.65 & 157.04 & Yes & $0^{*}$ & $1.4^{*}$ & & & $155.7^{*}$ & $157.1^{*}$ & & \\
\hline $22 \mathrm{H}-7$ & 0.61 & 157.1 & 157.71 & 157.04 & 157.64 & Yes & $0^{*}$ & $0.61^{*}$ & & & $157.1^{*}$ & $157.71^{*}$ & & \\
\hline $22 \mathrm{H}-\mathrm{CC}$ & 0.16 & 157.71 & 157.87 & 157.64 & 157.8 & Yes & $0^{*}$ & $0.16^{*}$ & & & $157.71^{*}$ & $157.87^{*}$ & & \\
\hline $23 \mathrm{H}-1$ & 1.31 & 157.8 & 159.11 & 157.8 & 158.04 & Yes & $0^{\dagger}$ & $0.14^{\dagger}$ & & & $157.8^{\dagger}$ & $157.94^{\dagger}$ & & \\
\hline $23 \mathrm{H}-1$ & 1.31 & 157.8 & 159.11 & 157.8 & 158.04 & Yes & 1.03 & $1.31^{*}$ & & & $158.83^{*}$ & $159.11^{*}$ & & \\
\hline $23 \mathrm{H}-\mathrm{CC}$ & 0.2 & 159.11 & 159.31 & 158.04 & 158.07 & Yes & $0^{*}$ & $0.2^{*}$ & & & $159.11^{*}$ & $159.31^{*}$ & & \\
\hline $24 \mathrm{H}-1$ & 1.5 & 159.3 & 160.8 & 159.3 & 160.78 & Yes & $0^{\dagger}$ & $0.13^{\dagger}$ & & & $159.3^{\dagger}$ & $159.43^{\dagger}$ & & \\
\hline $24 \mathrm{H}-3$ & 1.22 & 162.32 & 163.54 & 162.28 & 163.49 & Yes & $0^{*}$ & $1.22^{*}$ & & & $162.32^{\star}$ & $163.54^{*}$ & & \\
\hline $24 \mathrm{H}-4$ & 1.49 & 163.54 & 165.03 & 163.49 & 164.96 & Yes & $0^{*}$ & $1.49^{*}$ & & & $163.54^{*}$ & $165.03^{*}$ & & \\
\hline $24 \mathrm{H}-5$ & 1.28 & 165.03 & 166.31 & 164.96 & 166.22 & Yes & $0^{*}$ & $1.28^{*}$ & & & $165.03^{*}$ & $166.31^{*}$ & & \\
\hline $24 \mathrm{H}-\mathrm{CC}$ & 0.18 & 166.31 & 166.49 & 166.22 & 166.4 & Yes & $0^{*}$ & $0.18^{*}$ & & & $166.31^{*}$ & $166.49^{*}$ & & \\
\hline $26 \mathrm{H}-1$ & 0.5 & 173.3 & 173.8 & 173.3 & 173.34 & Yes & $0^{*}$ & $0.5^{*}$ & & & $173.3^{*}$ & $173.8^{*}$ & & \\
\hline $26 \mathrm{H}-\mathrm{CC}$ & 0.05 & 173.8 & 173.85 & 173.76 & 173.8 & Yes & $0^{*}$ & $0.05^{*}$ & & & $173.8^{*}$ & $173.85^{*}$ & & \\
\hline $27 \mathrm{H}-1$ & 1.5 & 173.8 & 175.3 & 173.8 & 175.3 & Yes & $0^{\dagger}$ & $0.25^{\dagger}$ & $0^{\dagger}$ & $1.5^{\dagger}$ & $173.8^{\dagger}$ & $174.05^{\dagger}$ & $174.05^{\dagger}$ & $175.3^{\dagger}$ \\
\hline $27 \mathrm{H}-2$ & 1.44 & 175.3 & 176.74 & 175.3 & 176.74 & Yes & $0.94^{*}$ & $1.44^{*}$ & $0^{\dagger}$ & $0.22^{\dagger}$ & $176.24^{\star}$ & $176.74^{*}$ & $175.3^{\dagger}$ & $175.52^{\dagger}$ \\
\hline $27 \mathrm{H}-3$ & 1.45 & 176.74 & 178.19 & 175.77 & 176.74 & Yes & $0^{*}$ & $1.45^{*}$ & & & $176.74^{*}$ & 178.19* & & \\
\hline
\end{tabular}


Table T1 (continued). (Continued on next page.)

\begin{tabular}{|c|c|c|c|c|c|c|c|c|c|c|c|c|c|c|}
\hline \multirow{2}{*}{$\begin{array}{l}\text { Core, } \\
\text { section }\end{array}$} & \multirow{2}{*}{$\begin{array}{l}\text { Recovered } \\
\text { length } \\
\text { (m) }\end{array}$} & \multicolumn{2}{|c|}{ Depth CSF-A (m) } & \multicolumn{2}{|c|}{ Depth CSF-B (m) } & \multirow{2}{*}{$\begin{array}{l}\text { Partial } \\
\text { stroke }\end{array}$} & \multicolumn{2}{|c|}{$\begin{array}{c}\text { Fall-in }^{*} \text { or flow-in } \\
\text { depth in section }(\mathrm{m}) \\
\end{array}$} & \multicolumn{2}{|c|}{$\begin{array}{l}\text { Possible fall-in* or } \\
\text { flow-in }{ }^{\dagger} \text { depth } \\
\text { in section }(\mathrm{m})\end{array}$} & \multicolumn{2}{|c|}{$\begin{array}{l}\text { Fall-in* or flow-in }^{\dagger} \\
\text { depth in hole } \\
\text { CSF-A }(m) \\
\end{array}$} & $\begin{array}{c}\text { Possible } \\
\text { flow-in } \\
\text { in hole C } \\
\end{array}$ & $\begin{array}{l}\text { fall-in* or } \\
\text { depth } \\
\text { SF-A (m) }\end{array}$ \\
\hline & & Top & Bottom & Top & Bottom & & Top & Bottom & Top & Bottom & Top & Bottom & Top & Bottom \\
\hline $27 \mathrm{H}-4$ & 1.4 & 178.19 & 179.59 & 177.13 & 178.19 & Yes & $0^{*}$ & $1.4^{*}$ & & & 178.19* & 179.59* & & \\
\hline $27 \mathrm{H}-5$ & 1.51 & 179.59 & 181.1 & 178.39 & 179.59 & Yes & $0^{*}$ & $1.51^{*}$ & & & $179.59^{*}$ & $181.1^{*}$ & & \\
\hline $27 \mathrm{H}-6$ & 1.22 & 181.1 & 182.32 & 180.06 & 181.1 & Yes & $0^{*}$ & $1.22^{*}$ & & & $181.1^{*}$ & $182.32^{*}$ & & \\
\hline $27 \mathrm{H}-7$ & 0.65 & 182.32 & 182.97 & 181.72 & 182.32 & Yes & $0^{*}$ & $0.65^{*}$ & & & $182.32^{*}$ & $182.97^{*}$ & & \\
\hline $27 \mathrm{H}-\mathrm{CC}$ & 0.09 & 182.97 & 183.06 & 182.88 & 182.97 & Yes & $0^{*}$ & $0.09^{*}$ & & & $182.97^{*}$ & $183.06^{*}$ & & \\
\hline $340-U 1400$ & & & & & & & & & & & & & & \\
\hline $2 \mathrm{H}-1$ & 1.28 & 3.5 & 4.78 & 3.5 & 4.78 & Yes & $0^{\dagger}$ & $0.35^{\dagger}$ & $0.35^{\dagger}$ & $1.28^{\dagger}$ & $3.5^{\dagger}$ & $3.85^{\dagger}$ & $3.85^{\dagger}$ & $4.78^{\dagger}$ \\
\hline $2 \mathrm{H}-2$ & 1.27 & 4.78 & 6.05 & 4.78 & 6.05 & Yes & & & $0^{\dagger}$ & $0.07^{\dagger}$ & & & $4.78^{\dagger}$ & 4.85 \\
\hline $2 \mathrm{H}-2$ & 1.27 & 4.78 & 6.05 & 4.78 & 6.05 & Yes & & & 0.54 & 1.27 & & & $5.32^{\star}$ & $6.05^{*}$ \\
\hline $2 \mathrm{H}-3$ & 0.86 & 6.05 & 6.91 & 5.41 & 6.05 & Yes & $0.55^{*}$ & $0.86^{*}$ & 0 & 0.55 & $6.6^{*}$ & $6.91^{*}$ & $6.05^{*}$ & $6.6^{*}$ \\
\hline $2 \mathrm{H}-4$ & 0.98 & 6.91 & 7.89 & 6.15 & 6.91 & Yes & $0^{*}$ & $0.98^{*}$ & & & $6.91^{*}$ & $7.89^{*}$ & & \\
\hline $2 \mathrm{H}-5$ & 1.32 & 7.89 & 9.21 & 6.88 & 7.89 & Yes & $0^{*}$ & $1.32^{*}$ & & & $7.89^{*}$ & $9.21^{*}$ & & \\
\hline $2 \mathrm{H}-6$ & 1.45 & 9.21 & 10.66 & 8.05 & 9.21 & Yes & $0^{*}$ & $1.45^{*}$ & & & $9.21^{*}$ & $10.66^{*}$ & & \\
\hline $2 \mathrm{H}-\mathrm{CC}$ & 0.09 & 10.66 & 10.75 & 10.57 & 10.66 & Yes & $0^{*}$ & $0.09^{*}$ & & & $10.66^{*}$ & $10.75^{*}$ & & \\
\hline $3 \mathrm{H}-6$ & 1.16 & 17.34 & 18.5 & 16.35 & 17.34 & Yes & $0.86^{*}$ & $1.16^{*}$ & & & $18.2^{\star}$ & $18.5^{\star}$ & & \\
\hline $3 \mathrm{H}-7$ & 0.61 & 18.5 & 19.11 & 17.93 & 18.5 & Yes & $0^{*}$ & $0.61^{*}$ & & & $18.5^{*}$ & $19.11^{*}$ & & \\
\hline $3 \mathrm{H}-\mathrm{CC}$ & 0.13 & 19.11 & 19.24 & 18.98 & 19.11 & Yes & $0^{*}$ & $0.13^{*}$ & & & $19.11^{*}$ & $19.24^{*}$ & & \\
\hline $4 \mathrm{H}-1$ & 1.42 & 19.2 & 20.62 & 19.2 & 20.35 & Yes & $0^{\dagger}$ & $0.19^{\dagger}$ & & & $19.2^{\dagger}$ & $19.39^{\dagger}$ & & \\
\hline $4 \mathrm{H}-5$ & 1.07 & 23.6 & 24.67 & 22.74 & 23.6 & Yes & $0.28^{*}$ & $1.07^{*}$ & & & $23.88^{*}$ & $24.67^{*}$ & & \\
\hline $4 \mathrm{H}-6$ & 1.36 & 24.67 & 26.03 & 23.58 & 24.67 & Yes & $0^{*}$ & $1.36^{*}$ & & & $24.67^{*}$ & $26.03^{*}$ & & \\
\hline $4 \mathrm{H}-\mathrm{CC}$ & 0.14 & 26.03 & 26.17 & 25.89 & 26.03 & Yes & $0^{*}$ & $0.14^{\star}$ & & & $26.03^{*}$ & $26.17^{*}$ & & \\
\hline $5 \mathrm{H}-1$ & 0.98 & 26.1 & 27.08 & 26.1 & 27.07 & Yes & $0^{\dagger}$ & $0.08^{\dagger}$ & & & $26.1^{\dagger}$ & $26.18^{\dagger}$ & & \\
\hline $5 \mathrm{H}-2$ & 1.28 & 27.08 & 28.36 & 27.08 & 28.36 & Yes & $0.09^{*}$ & $1.28^{*}$ & & & $27.17^{*}$ & $28.36^{*}$ & & \\
\hline $5 \mathrm{H}-3$ & 1.12 & 28.36 & 29.48 & 27.61 & 28.36 & Yes & $0^{*}$ & $1.12^{*}$ & & & $28.36^{*}$ & $29.48^{*}$ & & \\
\hline $5 \mathrm{H}-4$ & 1.52 & 29.48 & 31 & 28.43 & 29.48 & Yes & $0^{*}$ & $1.52^{*}$ & & & $29.48^{*}$ & $31^{*}$ & & \\
\hline $5 \mathrm{H}-5$ & 0.55 & 31 & 31.55 & 30.51 & 31 & Yes & $0^{*}$ & $0.55^{*}$ & & & $31^{*}$ & $31.55^{*}$ & & \\
\hline $5 \mathrm{H}-\mathrm{CC}$ & 0.32 & 31.55 & 31.87 & 31.25 & 31.55 & Yes & $0^{*}$ & $0.32^{*}$ & & & $31.55^{*}$ & $31.87^{*}$ & & \\
\hline $6 \mathrm{H}-1$ & 0.81 & 31.8 & 32.61 & 31.8 & 32.61 & Yes & $0^{\dagger}$ & $0.03^{\dagger}$ & & & $31.8^{\dagger}$ & $31.83^{\dagger}$ & & \\
\hline $6 \mathrm{H}-1$ & 0.81 & 31.8 & 32.61 & 31.8 & 32.61 & Yes & $0.22^{*}$ & $0.81^{*}$ & & & $32.02^{*}$ & $32.61^{*}$ & & \\
\hline $6 \mathrm{H}-2$ & 0.67 & 32.61 & 33.28 & 32.6 & 33.26 & Yes & $0^{*}$ & $0.67^{*}$ & & & $32.61^{*}$ & $33.28^{*}$ & & \\
\hline $6 \mathrm{H}-3$ & 1.18 & 33.28 & 34.46 & 33.26 & 34.42 & Yes & $0^{*}$ & $1.18^{*}$ & & & $33.28^{*}$ & $34.46^{*}$ & & \\
\hline $6 \mathrm{H}-4$ & 0.8 & 34.46 & 35.26 & 34.42 & 35.21 & Yes & $0^{*}$ & $0.8^{*}$ & & & $34.46^{*}$ & $35.26^{*}$ & & \\
\hline $6 \mathrm{H}-5$ & 0.99 & 35.26 & 36.25 & 35.26 & 36.25 & Yes & $0^{*}$ & $0.99^{*}$ & & & $35.26^{*}$ & $36.25^{*}$ & & \\
\hline $6 \mathrm{H}-6$ & 0.74 & 36.25 & 36.99 & 36.25 & 36.99 & Yes & $0^{*}$ & $0.74^{\star}$ & & & $36.25^{*}$ & $36.99^{*}$ & & \\
\hline $6 \mathrm{H}-7$ & 1.24 & 36.99 & 38.23 & 36.99 & 38.23 & Yes & $0^{*}$ & $1.24^{\star}$ & & & $36.99^{*}$ & $38.23^{*}$ & & \\
\hline $6 \mathrm{H}-\mathrm{CC}$ & 0.07 & 38.23 & 38.3 & 38.23 & 38.3 & Yes & $0^{*}$ & $0.07^{*}$ & & & $38.23^{*}$ & $38.3^{*}$ & & \\
\hline $7 \mathrm{H}-1$ & 1.31 & 38.3 & 39.61 & 38.3 & 38.92 & Yes & $0^{*}$ & $1.31^{*}$ & & & $38.3^{*}$ & $39.61^{*}$ & & \\
\hline $7 \mathrm{H}-2$ & 1.01 & 39.61 & 40.62 & 39.61 & 40.62 & Yes & $0^{*}$ & $1.01^{*}$ & & & $39.61^{*}$ & $40.62^{\star}$ & & \\
\hline $7 \mathrm{H}-3$ & 0.65 & 40.62 & 41.27 & 40.11 & 40.62 & Yes & $0^{*}$ & $0.65^{*}$ & & & $40.62^{*}$ & $41.27^{\star}$ & & \\
\hline 7H-CC & 0.17 & 41.27 & 41.44 & 41.11 & 41.27 & Yes & $0^{*}$ & $0.17^{\star}$ & & & $41.27^{*}$ & $41.44^{*}$ & & \\
\hline $8 \mathrm{H}-1$ & 1.4 & 41.4 & 42.8 & 41.4 & 42.61 & Yes & $0^{\dagger}$ & $0.25^{\dagger}$ & & & $41.4^{\dagger}$ & $41.65^{\dagger}$ & & \\
\hline $8 \mathrm{H}-1$ & 1.4 & 41.4 & 42.8 & 41.4 & 42.61 & Yes & $0.25^{*}$ & $1.4^{\star}$ & & & $41.65^{*}$ & $42.8^{*}$ & & \\
\hline $8 \mathrm{H}-2$ & 0.3 & 42.8 & 43.1 & 42.8 & 43.1 & Yes & $0^{*}$ & $0.3^{*}$ & & & $42.8^{*}$ & $43.1^{*}$ & & \\
\hline $8 \mathrm{H}-3$ & 0.21 & 43.1 & 43.31 & 42.91 & 43.1 & Yes & $0^{*}$ & $0.21^{*}$ & & & $43.1^{*}$ & $43.31^{*}$ & & \\
\hline $8 \mathrm{H}-4$ & 1.26 & 43.31 & 44.57 & 42.55 & 43.31 & Yes & $0^{*}$ & $1.26^{*}$ & & & $43.31^{*}$ & $44.57^{*}$ & & \\
\hline $8 \mathrm{H}-5$ & 1.5 & 44.57 & 46.07 & 43.55 & 44.57 & Yes & $0^{*}$ & $1.5^{*}$ & & & $44.57^{\star}$ & $46.07^{*}$ & & \\
\hline $8 \mathrm{H}-6$ & 0.77 & 46.07 & 46.84 & 45.41 & 46.07 & Yes & $0^{*}$ & $0.77^{*}$ & & & $46.07^{*}$ & $46.84^{*}$ & & \\
\hline $8 \mathrm{H}-\mathrm{CC}$ & 0.21 & 46.84 & 47.05 & 46.64 & 46.84 & Yes & $0^{*}$ & $0.21^{*}$ & & & $46.84^{*}$ & $47.05^{*}$ & & \\
\hline $9 \mathrm{H}-1$ & 1.4 & 47 & 48.4 & 47 & 48.07 & Yes & $0^{*}$ & $1.4^{*}$ & & & $47^{*}$ & $48.4^{*}$ & & \\
\hline $9 \mathrm{H}-2$ & 1.22 & 48.4 & 49.62 & 48.4 & 49.62 & Yes & $0^{*}$ & $1.22^{*}$ & & & $48.4^{*}$ & $49.62^{*}$ & & \\
\hline $9 \mathrm{H}-3$ & 0.98 & 49.62 & 50.6 & 48.91 & 49.62 & Yes & $0^{*}$ & $0.98^{*}$ & & & $49.2^{*}$ & $50.6^{*}$ & & \\
\hline $9 \mathrm{H}-4$ & 0.64 & 50.6 & 51.24 & 50.06 & 50.6 & Yes & $0^{*}$ & $0.64^{*}$ & & & $50.6^{*}$ & $51.24^{*}$ & & \\
\hline 9H-CC & 0.15 & 51.24 & 51.39 & 51.1 & 51.24 & Yes & $0^{*}$ & $0.15^{*}$ & & & $51.24^{*}$ & $51.39^{*}$ & & \\
\hline $340-U 140$ & & & & & & & & & & & & & & \\
\hline $1 \mathrm{H}-2$ & 1.5 & 1.5 & 3 & 1.5 & 2.99 & No & $1.45^{*}$ & $1.5^{*}$ & & & $2.95^{*}$ & $3^{*}$ & & \\
\hline $1 \mathrm{H}-3$ & 1.5 & 3 & 4.5 & 2.99 & 4.49 & No & $0^{*}$ & $1.5^{*}$ & & & $3^{*}$ & $4.5^{*}$ & & \\
\hline $1 \mathrm{H}-4$ & 1.5 & 4.5 & 6 & 4.49 & 5.98 & No & $0^{*}$ & $1.5^{*}$ & & & $4.5^{\star}$ & $6^{*}$ & & \\
\hline $1 \mathrm{H}-5$ & 0.65 & 6 & 6.65 & 5.98 & 6.63 & No & $0^{*}$ & $0.65^{*}$ & & & $6^{*}$ & $6.65^{*}$ & & \\
\hline $1 \mathrm{H}-\mathrm{CC}$ & 0.17 & 6.65 & 6.82 & 6.63 & 6.8 & No & $0^{*}$ & $0.17^{*}$ & & & $6.65^{*}$ & $6.82^{*}$ & & \\
\hline $2 \mathrm{H}-1$ & 1.02 & 6.8 & 7.82 & 6.8 & 7.8 & Yes & $0^{*}$ & $1.02^{*}$ & & & $6.8^{*}$ & $7.82^{*}$ & & \\
\hline $2 \mathrm{H}-2$ & 0.61 & 7.82 & 8.43 & 7.8 & 8.4 & Yes & $0^{*}$ & $0.61^{*}$ & & & $7.82^{*}$ & $8.43^{*}$ & & \\
\hline $2 \mathrm{H}-\mathrm{CC}$ & 0.2 & 8.43 & 8.63 & 8.4 & 8.6 & Yes & $0^{*}$ & $0.2^{*}$ & & & $8.43^{*}$ & $8.63^{*}$ & & \\
\hline $3 \mathrm{H}-1$ & 0.81 & 8.6 & 9.41 & 8.6 & 9.41 & Yes & $0^{*}$ & $0.81^{*}$ & & & $8.6^{*}$ & $9.41^{*}$ & & \\
\hline $3 \mathrm{H}-2$ & 1 & 9.41 & 10.41 & 9.41 & 10.41 & Yes & $0^{*}$ & $1^{*}$ & & & $9.41^{*}$ & $10.41^{*}$ & & \\
\hline $3 \mathrm{H}-3$ & 0.58 & 10.41 & 10.99 & 10.41 & 10.99 & Yes & $0^{*}$ & $0.58^{*}$ & & & $10.41^{*}$ & $10.99^{*}$ & & \\
\hline $3 \mathrm{H}-\mathrm{CC}$ & 0.21 & 10.99 & 11.2 & 10.99 & 11.2 & Yes & $0^{*}$ & $0.21^{*}$ & & & $10.99^{*}$ & $11.2^{*}$ & & \\
\hline
\end{tabular}


Table T1 (continued). (Continued on next page.)

\begin{tabular}{|c|c|c|c|c|c|c|c|c|c|c|c|c|c|c|}
\hline \multirow{2}{*}{$\begin{array}{l}\text { Core, } \\
\text { section }\end{array}$} & \multirow{2}{*}{$\begin{array}{l}\text { Recovered } \\
\text { length } \\
\text { (m) }\end{array}$} & \multicolumn{2}{|c|}{ Depth CSF-A (m) } & \multicolumn{2}{|c|}{ Depth CSF-B (m) } & \multirow{2}{*}{$\begin{array}{l}\text { Partial } \\
\text { stroke }\end{array}$} & \multicolumn{2}{|c|}{$\begin{array}{c}\text { Fall-in* or flow-in } \\
\text { depth in section }(\mathrm{m}) \\
\end{array}$} & \multicolumn{2}{|c|}{$\begin{array}{l}\text { Possible fall-in* or } \\
\text { flow-in }{ }^{\dagger} \text { depth } \\
\text { in section }(\mathrm{m}) \\
\end{array}$} & $\begin{array}{r}\text { Fall-in* or } \\
\text { depth i } \\
\text { CSF-A }\end{array}$ & $\begin{array}{l}\text { flow-in } \\
\text { n hole } \\
\text { (m) }\end{array}$ & $\begin{array}{r}\text { Possible f } \\
\text { flow-in } \\
\text { in hole C } \\
\end{array}$ & $\begin{array}{l}\text { all-in* or } \\
\text { depth } \\
\text { SF-A (m) }\end{array}$ \\
\hline & & Top & Bottom & Top & Bottom & & Top & Bottom & Top & Bottom & Top & Bottom & Top & Bottom \\
\hline $4 \mathrm{H}-1$ & 0.91 & 11.2 & 12.11 & 11.2 & 12.1 & Yes & $0^{\dagger}$ & $0.07^{\dagger}$ & & & $11.2^{\dagger}$ & $11.27^{\dagger}$ & & \\
\hline $4 \mathrm{H}-1$ & 0.91 & 11.2 & 12.11 & 11.2 & 12.1 & Yes & $0.07^{*}$ & $0.91^{*}$ & & & $11.27^{*}$ & $12.11^{*}$ & & \\
\hline $4 \mathrm{H}-2$ & 0.9 & 12.11 & 13.01 & 12.1 & 12.98 & Yes & $0^{*}$ & $0.9^{*}$ & & & $12.11^{*}$ & $13.01^{*}$ & & \\
\hline $4 \mathrm{H}-3$ & 0.91 & 13.01 & 13.92 & 12.98 & 13.88 & Yes & $0^{*}$ & $0.91^{*}$ & & & $13.01^{*}$ & $13.92^{*}$ & & \\
\hline $4 \mathrm{H}-4$ & 1.5 & 13.92 & 15.42 & 13.88 & 15.36 & Yes & $0^{*}$ & $1.5^{*}$ & & & $13.92^{*}$ & $15.42^{*}$ & & \\
\hline $4 \mathrm{H}-5$ & 1.3 & 15.42 & 16.72 & 15.36 & 16.64 & Yes & $0^{*}$ & $1.3^{*}$ & & & $15.42^{*}$ & $16.72^{*}$ & & \\
\hline $4 \mathrm{H}-6$ & 0.57 & 16.72 & 17.29 & 16.64 & 17.2 & Yes & $0^{*}$ & $0.57^{*}$ & & & $16.72^{*}$ & $17.29^{*}$ & & \\
\hline $4 \mathrm{H}-\mathrm{CC}$ & 0.2 & 17.29 & 17.49 & 17.2 & 17.4 & Yes & $0^{*}$ & $0.2^{*}$ & & & $17.29^{*}$ & $17.49^{*}$ & & \\
\hline $5 \mathrm{H}-1$ & 0.81 & 17.4 & 18.21 & 17.4 & 18.18 & Yes & $0^{*}$ & $0.81^{*}$ & & & $17.4^{*}$ & $18.21^{*}$ & & \\
\hline $5 \mathrm{H}-2$ & 1.39 & 18.21 & 19.6 & 18.18 & 19.53 & Yes & $0^{*}$ & $1.39^{*}$ & & & $18.21^{*}$ & $19.6^{*}$ & & \\
\hline $5 \mathrm{H}-\mathrm{CC}$ & 0.07 & 19.6 & 19.67 & 19.53 & 19.6 & Yes & $0^{*}$ & $0.07^{*}$ & & & $19.6^{*}$ & $19.67^{*}$ & & \\
\hline $6 \mathrm{H}-1$ & 1.24 & 19.6 & 20.84 & 19.6 & 20.84 & Yes & $0^{\dagger}$ & $0.55^{\dagger}$ & & & $19.6^{\dagger}$ & $20.15^{\dagger}$ & & \\
\hline $6 \mathrm{H}-1$ & 1.24 & 19.6 & 20.84 & 19.6 & 20.84 & Yes & $0.55^{*}$ & $1.24^{*}$ & & & $20.15^{*}$ & $20.84^{*}$ & & \\
\hline $6 \mathrm{H}-2$ & 1.5 & 20.84 & 22.34 & 20.84 & 22.33 & Yes & $0^{*}$ & $1.5^{*}$ & & & $20.84^{*}$ & $22.34^{*}$ & & \\
\hline $6 \mathrm{H}-3$ & 1.5 & 22.34 & 23.84 & 22.33 & 23.83 & Yes & $0^{*}$ & $1.5^{*}$ & & & $22.34^{*}$ & $23.84^{*}$ & & \\
\hline $6 \mathrm{H}-4$ & 1.48 & 23.84 & 25.32 & 23.83 & 25.3 & Yes & $0^{*}$ & $1.48^{\star}$ & & & $23.84^{*}$ & $25.32^{*}$ & & \\
\hline $6 \mathrm{H}-\mathrm{CC}$ & 0.2 & 25.32 & 25.52 & 25.3 & 25.5 & Yes & $0^{*}$ & $0.2^{*}$ & & & $25.32^{*}$ & $25.52^{*}$ & & \\
\hline $9 \mathrm{H}-3$ & 0.9 & 47.45 & 48.35 & 47.44 & 48.34 & Yes & $0.53^{*}$ & $0.9^{*}$ & & & $47.98^{*}$ & $48.35^{*}$ & & \\
\hline $9 \mathrm{H}-\mathrm{CC}$ & 0.16 & 48.35 & 48.51 & 48.34 & 48.5 & Yes & $0^{*}$ & $0.16^{*}$ & & & $48.35^{*}$ & $48.51^{*}$ & & \\
\hline $12 \mathrm{H}-2$ & 1.21 & 68.97 & 70.18 & 68.97 & 70.18 & Yes & $0.94^{\star}$ & $1.21^{\star}$ & & & $69.91^{*}$ & $70.18^{*}$ & & \\
\hline $12 \mathrm{H}-3$ & 0.28 & 70.18 & 70.46 & 70.18 & 70.46 & Yes & $0^{*}$ & $0.28^{\star}$ & & & $70.18^{*}$ & $70.46^{*}$ & & \\
\hline $12 \mathrm{H}-4$ & 1.17 & 70.46 & 71.63 & 70.46 & 71.63 & Yes & $0^{*}$ & $1.17^{*}$ & & & $70.46^{*}$ & $71.63^{*}$ & & \\
\hline $12 \mathrm{H}-5$ & 1.5 & 71.63 & 73.13 & 71.63 & 73.13 & Yes & $0^{*}$ & $1.5^{\star}$ & & & $71.63^{*}$ & $73.13^{*}$ & & \\
\hline $12 \mathrm{H}-6$ & 0.69 & 73.13 & 73.82 & 73.13 & 73.82 & Yes & $0^{*}$ & $0.69^{*}$ & & & $73.13^{*}$ & $73.82^{*}$ & & \\
\hline $12 \mathrm{H}-\mathrm{CC}$ & 0.18 & 73.82 & 74 & 73.82 & 74 & Yes & $0^{*}$ & $0.18^{*}$ & & & $73.82^{*}$ & $74^{*}$ & & \\
\hline $15 \mathrm{H}-6$ & 0.96 & 100.38 & 101.34 & 100.38 & 101.34 & No & & & $0.04^{*}$ & $0.96^{*}$ & & & $100.42^{*}$ & $101.34^{*}$ \\
\hline $15 \mathrm{H}-7$ & 0.65 & 101.34 & 101.99 & 101.34 & 101.99 & No & 0.12 & $0.65^{*}$ & $0^{*}$ & $0.12^{*}$ & $101.46^{*}$ & 101.99* & $101.34^{*}$ & $101.46^{*}$ \\
\hline $15 \mathrm{H}-\mathrm{CC}$ & 0.17 & 101.99 & 102.16 & 101.99 & 102.16 & No & $0^{*}$ & $0.17^{*}$ & & & 101.99* & $102.16^{*}$ & & \\
\hline $16 \mathrm{H}-1$ & 1.34 & 102.5 & 103.84 & 102.5 & 103.83 & Yes & $0^{*}$ & $1.34^{*}$ & & & $102.5^{*}$ & $103.84^{*}$ & & \\
\hline $16 \mathrm{H}-2$ & 1.01 & 103.84 & 104.85 & 103.83 & 104.83 & Yes & $0^{*}$ & $1.01^{*}$ & & & $103.84^{*}$ & $104.85^{*}$ & & \\
\hline $16 \mathrm{H}-3$ & 0.72 & 104.85 & 105.57 & 104.83 & 105.54 & Yes & $0^{*}$ & $0.72^{*}$ & & & $104.85^{*}$ & $105.57^{*}$ & & \\
\hline $16 \mathrm{H}-\mathrm{CC}$ & 0.16 & 105.57 & 105.73 & 105.54 & 105.7 & Yes & $0^{*}$ & $0.16^{*}$ & & & $105.57^{*}$ & $105.73^{*}$ & & \\
\hline $20 \mathrm{H}-1$ & 1.5 & 128.8 & 130.3 & 128.8 & 130.29 & No & & & $0^{\dagger}$ & $1.5^{\dagger}$ & & & $128.8^{\dagger}$ & $130.3^{\dagger}$ \\
\hline $20 \mathrm{H}-2$ & 1.51 & 130.3 & 131.81 & 130.29 & 131.78 & No & & & $0^{\dagger}$ & $0.67^{\dagger}$ & & & $130.3^{\dagger}$ & $130.97^{\dagger}$ \\
\hline $24 \mathrm{H}-1$ & 1.47 & 165.1 & 166.57 & 165.1 & 166.57 & No & & & $0^{*}$ & $1.25^{*}$ & & & $165.1^{*}$ & $166.35^{*}$ \\
\hline 340-U1400 & & & & & & & & & & & & & & \\
\hline $2 \mathrm{H}-1$ & 1.5 & 15 & 16.5 & 15 & 16.48 & Yes & $0^{\dagger}$ & $1.5^{\dagger}$ & & & $15^{\dagger}$ & $16.5^{\dagger}$ & & \\
\hline $2 \mathrm{H}-2$ & 1.5 & 16.5 & 18 & 16.48 & 17.96 & Yes & $0^{\dagger}$ & $1.5^{\dagger}$ & & & $16.5^{\dagger}$ & $18^{\dagger}$ & & \\
\hline $2 \mathrm{H}-3$ & 0.93 & 18 & 18.93 & 17.96 & 18.88 & Yes & $0^{\dagger}$ & $0.15^{\dagger}$ & & & $18^{\dagger}$ & $18.15^{\dagger}$ & & \\
\hline $2 \mathrm{H}-3$ & 0.93 & 18 & 18.93 & 17.96 & 18.88 & Yes & $0.15^{*}$ & $0.93^{*}$ & & & $18.15^{*}$ & $18.93^{*}$ & & \\
\hline $2 \mathrm{H}-4$ & 1.19 & 18.93 & 20.12 & 18.88 & 20.05 & Yes & $0^{*}$ & $1.19^{*}$ & & & $18.93^{*}$ & $20.12^{*}$ & & \\
\hline $2 \mathrm{H}-5$ & 1.5 & 20.12 & 21.62 & 20.05 & 21.53 & Yes & $0^{*}$ & $1.5^{*}$ & & & $20.12^{*}$ & $21.62^{*}$ & & \\
\hline $2 \mathrm{H}-\mathrm{CC}$ & 0.07 & 21.62 & 21.69 & 21.53 & 21.6 & Yes & $0^{*}$ & $0.07^{\star}$ & & & $21.62^{*}$ & $21.69^{*}$ & & \\
\hline $3 \mathrm{H}-1$ & 1.5 & 21.6 & 23.1 & 21.6 & 23.08 & Yes & $0^{\dagger}$ & $0.47^{\dagger}$ & & & $21.6^{\dagger}$ & $22.07^{\dagger}$ & & \\
\hline $3 \mathrm{H}-3$ & 1.51 & 24.6 & 26.11 & 24.56 & 26.06 & Yes & 0.25 & $1.51^{*}$ & & & $24.85^{*}$ & $26.11^{*}$ & & \\
\hline $3 \mathrm{H}-4$ & 1.5 & 26.11 & 27.61 & 26.06 & 27.54 & Yes & $0^{*}$ & $0.9^{*}$ & & & $26.11^{*}$ & $27.01^{*}$ & & \\
\hline $3 \mathrm{H}-6$ & 1.04 & 28.19 & 29.23 & 28.11 & 29.14 & Yes & $0^{*}$ & $1.04^{\star}$ & & & $28.19^{*}$ & $29.23^{*}$ & & \\
\hline $3 \mathrm{H}-\mathrm{CC}$ & 0.16 & 29.23 & 29.39 & 29.14 & 29.3 & Yes & $0^{*}$ & $0.16^{*}$ & & & $29.23^{*}$ & $29.39^{*}$ & & \\
\hline $4 \mathrm{H}-1$ & 1.5 & 29.3 & 30.8 & 29.3 & 30.76 & No & $0^{\dagger}$ & $0.15^{\dagger}$ & & & $29.3^{\dagger}$ & $29.45^{\dagger}$ & & \\
\hline $5 \mathrm{H}-1$ & 1.45 & 38.8 & 40.25 & 38.8 & 40.25 & No & $0^{\dagger}$ & $0.84^{\dagger}$ & & & $38.8^{\dagger}$ & $39.64^{\dagger}$ & & \\
\hline $7 \mathrm{H}-1$ & 1.5 & 57.8 & 59.3 & 57.8 & 59.3 & Yes & $0^{\dagger}$ & $1.23^{\dagger}$ & & & $57.8^{\dagger}$ & $59.03^{\dagger}$ & & \\
\hline $8 \mathrm{H}-1$ & 1.41 & 66.6 & 68.01 & 66.6 & 67.93 & No & & & $0^{\dagger}$ & $0.97^{\dagger}$ & & & $66.6^{\dagger}$ & $67.57^{\dagger}$ \\
\hline $8 \mathrm{H}-2$ & 1.5 & 68.01 & 69.51 & 67.93 & 69.35 & No & & & $0.63^{*}$ & $1.5^{*}$ & & & $68.64^{*}$ & $69.51^{*}$ \\
\hline $8 \mathrm{H}-3$ & 1.52 & 69.51 & 71.03 & 69.35 & 70.78 & No & & & $0^{*}$ & $1.52^{\star}$ & & & $69.51^{*}$ & $71.03^{*}$ \\
\hline $8 \mathrm{H}-4$ & 1.51 & 71.03 & 72.54 & 70.78 & 72.21 & No & & & $0^{*}$ & $1.51^{*}$ & & & $71.03^{*}$ & $72.54^{*}$ \\
\hline $8 \mathrm{H}-5$ & 1.51 & 72.54 & 74.05 & 72.21 & 73.64 & No & & & $0^{*}$ & $1.51^{*}$ & & & $72.54^{*}$ & $74.05^{*}$ \\
\hline $8 \mathrm{H}-6$ & 1.51 & 74.05 & 75.56 & 73.64 & 75.06 & No & & & $0^{*}$ & $1.51^{*}$ & & & $74.05^{*}$ & $75.56^{*}$ \\
\hline $8 \mathrm{H}-7$ & 0.7 & 75.56 & 76.26 & 75.06 & 75.72 & No & & & $0^{*}$ & $0.7^{*}$ & & & $75.56^{*}$ & $76.26^{*}$ \\
\hline $8 \mathrm{H}-\mathrm{CC}$ & 0.4 & 76.26 & 76.66 & 75.72 & 76.1 & No & & & $0^{*}$ & $0.4^{*}$ & & & $76.26^{*}$ & $76.66^{*}$ \\
\hline $9 \mathrm{H}-1$ & 1.5 & 76.1 & 77.6 & 76.1 & 77.58 & Yes & $0^{\dagger}$ & $0.16^{\dagger}$ & & & $76.1^{\dagger}$ & $76.26^{\dagger}$ & & \\
\hline $10 \mathrm{H}-\mathrm{CC}$ & 0.26 & 84.63 & 84.89 & 84.54 & 84.8 & Yes & $0^{*}$ & $0.26^{*}$ & & & $84.63^{*}$ & $84.89^{*}$ & & \\
\hline $13 \mathrm{H}-6$ & 0.48 & 110.83 & 111.31 & 110.79 & 111.27 & Yes & & & $0.23^{*}$ & $0.48^{*}$ & & & $111.06^{*}$ & $111.31^{*}$ \\
\hline $13 \mathrm{H}-\mathrm{CC}$ & 0.23 & 111.31 & 111.54 & 111.27 & 111.5 & Yes & & & $0^{*}$ & $0.23^{*}$ & & & $111.31^{*}$ & $111.54^{*}$ \\
\hline $18 \mathrm{H}-1$ & 1.5 & 143.6 & 145.1 & 143.6 & 145.1 & No & & & $0^{\dagger}$ & $1.38^{\dagger}$ & & & $143.6^{\dagger}$ & $144.98^{\dagger}$ \\
\hline $340-U 1401$ & & & & & & & & & & & & & & \\
\hline $1 \mathrm{H}-6$ & 0.75 & 6.81 & 7.56 & 6.77 & 7.51 & Yes & & & $0.69^{*}$ & $0.75^{*}$ & & & $7.5^{*}$ & $7.56^{\star}$ \\
\hline $1 \mathrm{H}-\mathrm{CC}$ & 0.19 & 7.56 & 7.75 & 7.51 & 7.7 & Yes & & & $0^{*}$ & $0.19^{*}$ & & & $7.56^{*}$ & $7.75^{*}$ \\
\hline
\end{tabular}


Table T1 (continued).

\begin{tabular}{|c|c|c|c|c|c|c|c|c|c|c|c|c|c|c|}
\hline \multirow{2}{*}{$\begin{array}{c}\text { Core, } \\
\text { section }\end{array}$} & \multirow{2}{*}{$\begin{array}{l}\text { Recovered } \\
\text { length } \\
(\mathrm{m})\end{array}$} & \multicolumn{2}{|c|}{ Depth CSF-A (m) } & \multicolumn{2}{|c|}{ Depth CSF-B (m) } & \multirow{2}{*}{$\begin{array}{l}\text { Partial } \\
\text { stroke }\end{array}$} & \multicolumn{2}{|c|}{$\begin{array}{c}\text { Fall-in* or flow-in }{ }^{\dagger} \\
\text { depth in section }(m)\end{array}$} & \multicolumn{2}{|c|}{$\begin{array}{l}\text { Possible fall-in* or } \\
\text { flow-in }{ }^{\dagger} \text { depth } \\
\text { in section }(\mathrm{m})\end{array}$} & \multicolumn{2}{|c|}{$\begin{array}{c}\text { Fall-in* or flow-in }{ }^{\dagger} \\
\text { depth in hole } \\
\text { CSF-A (m) }\end{array}$} & \multicolumn{2}{|c|}{$\begin{array}{l}\text { Possible fall-in* or } \\
\text { flow-in }{ }^{\dagger} \text { depth } \\
\text { in hole CSF-A (m) }\end{array}$} \\
\hline & & Top & Bottom & Top & Bottom & & Top & Bottom & Top & Bottom & Top & Bottom & Top & Bottom \\
\hline $2 \mathrm{H}-1$ & 1.5 & 7.7 & 9.2 & 7.7 & 9.19 & Yes & & & $0^{\dagger}$ & $0.2^{\dagger}$ & & & $7.7^{\dagger}$ & $7.9^{\dagger}$ \\
\hline $2 \mathrm{H}-2$ & 1.5 & 9.2 & 10.7 & 9.19 & 10.68 & Yes & & & $0.66^{*}$ & $1.5^{*}$ & & & $9.86^{*}$ & $10.7^{*}$ \\
\hline $2 \mathrm{H}-3$ & 1.2 & 10.7 & 11.9 & 10.68 & 11.87 & Yes & & & $0^{*}$ & $1.2^{*}$ & & & $10.7^{*}$ & $11.9^{*}$ \\
\hline $2 \mathrm{H}-4$ & 0.49 & 11.9 & 12.39 & 11.87 & 12.36 & Yes & & & $0^{*}$ & $0.49^{*}$ & & & $11.9^{*}$ & $12.39^{*}$ \\
\hline $2 \mathrm{H}-\mathrm{CC}$ & 0.14 & 12.39 & 12.53 & 12.36 & 12.5 & Yes & & & $0^{*}$ & $0.14^{*}$ & & & $12.39^{*}$ & $12.53^{*}$ \\
\hline $3 \mathrm{H}-1$ & 1.03 & 12.5 & 13.53 & 12.5 & 13.51 & Yes & $0^{*}$ & $1.03^{*}$ & & & $12.5^{*}$ & $13.53^{*}$ & & \\
\hline $3 \mathrm{H}-\mathrm{CC}$ & 0.19 & 13.53 & 13.72 & 13.51 & 13.7 & Yes & $0^{*}$ & $0.19^{*}$ & & & $13.53^{*}$ & $13.72^{*}$ & & \\
\hline $4 \mathrm{H}-1$ & 0.45 & 13.7 & 14.15 & 13.7 & 14.12 & Yes & $0^{*}$ & $0.45^{*}$ & & & $13.7^{\star}$ & $14.15^{*}$ & & \\
\hline $4 \mathrm{H}-\mathrm{CC}$ & 0.2 & 14.15 & 14.35 & 14.12 & 14.3 & Yes & $0 *$ & $0.2^{*}$ & & & $14.15^{*}$ & $14.35^{*}$ & & \\
\hline \multicolumn{15}{|c|}{ 340-U1401B- } \\
\hline $1 \mathrm{H}-5$ & 1.44 & 6 & 7.44 & 5.93 & 7.35 & Yes & & & $1.05^{*}$ & $1.44^{*}$ & & & $7.05^{*}$ & $7.44^{*}$ \\
\hline $1 \mathrm{H}-\mathrm{CC}$ & 0.25 & 7.44 & 7.69 & 7.35 & 7.6 & Yes & & & $0^{*}$ & $0.25^{*}$ & & & $7.44^{*}$ & $7.69 *$ \\
\hline $2 \mathrm{H}-1$ & 1.5 & 7.6 & 9.1 & 7.6 & 9.1 & Yes & & & $0^{\dagger}$ & $1.5^{\dagger}$ & & & $7.6^{\dagger}$ & $9.1^{\dagger}$ \\
\hline $2 \mathrm{H}-2$ & 0.88 & 9.1 & 9.98 & 9.1 & 9.98 & Yes & & & $0^{\dagger}$ & $0.5^{\dagger}$ & & & $9.1^{\dagger}$ & $9.6^{\dagger}$ \\
\hline $2 \mathrm{H}-4$ & 0.58 & 10.99 & 11.57 & 10.99 & 11.57 & Yes & $0^{*}$ & $0.58^{*}$ & & & $10.99^{*}$ & $11.57^{*}$ & & \\
\hline $2 \mathrm{H}-5$ & 0.5 & 11.57 & 12.07 & 11.57 & 12.07 & Yes & $0^{*}$ & $0.5^{*}$ & & & $11.57^{*}$ & $12.07^{*}$ & & \\
\hline $2 \mathrm{H}-\mathrm{CC}$ & 0.13 & 12.07 & 12.2 & 12.07 & 12.2 & Yes & 0 * & $0.13^{*}$ & & & $12.07^{*}$ & $12.2^{*}$ & & \\
\hline $3 \mathrm{H}-\mathrm{CC}$ & 0.13 & 12.8 & 12.93 & 12.8 & 12.9 & Yes & $0^{\dagger}$ & $0.13^{\dagger}$ & & & $12.8^{\dagger}$ & $12.93^{\dagger}$ & & \\
\hline \multicolumn{15}{|c|}{ 340-U1401C- } \\
\hline $1 \mathrm{H}-4$ & 1.5 & 4.44 & 5.94 & 4.42 & 5.92 & Yes & & & $1.25^{*}$ & $1.5^{*}$ & & & $5.69^{*}$ & $5.94^{*}$ \\
\hline $1 \mathrm{H}-5$ & 1.2 & 5.94 & 7.14 & 5.92 & 7.11 & Yes & & & $0^{*}$ & $1.2^{*}$ & & & $5.94^{*}$ & $7.14^{*}$ \\
\hline $1 \mathrm{H}-6$ & 0.61 & 7.14 & 7.75 & 7.11 & 7.72 & Yes & & & 0 * & $0.61^{*}$ & & & $7.14^{*}$ & $7.75^{*}$ \\
\hline $1 \mathrm{H}-\mathrm{CC}$ & 0.28 & 7.75 & 8.03 & 7.72 & 8 & Yes & & & $0^{*}$ & $0.28^{*}$ & & & $7.75^{*}$ & $8.03^{*}$ \\
\hline $2 \mathrm{H}-1$ & 0.65 & 8 & 8.65 & 8 & 8.64 & Yes & $0^{\dagger}$ & $0.65^{\dagger}$ & & & $8^{\dagger}$ & $8.65^{\dagger}$ & & \\
\hline $2 \mathrm{H}-2$ & 0.68 & 8.65 & 9.33 & 8.64 & 9.3 & Yes & $0^{\dagger}$ & $0.68^{\dagger}$ & & & $8.65^{\dagger}$ & $9.33^{\dagger}$ & & \\
\hline $2 \mathrm{H}-\mathrm{CC}$ & 0.1 & 9.33 & 9.43 & 9.3 & 9.4 & Yes & $0^{\dagger}$ & $0.1^{\dagger}$ & & & $9.33^{\dagger}$ & $9.43^{\dagger}$ & & \\
\hline $3 \mathrm{H}-1$ & 0.87 & 9.4 & 10.27 & 9.4 & 10.2 & Yes & $0^{\dagger}$ & $0.87^{\dagger}$ & & & $9.4^{\dagger}$ & $10.27^{\dagger}$ & & \\
\hline $3 \mathrm{H}-\mathrm{CC}$ & 0.11 & 10.27 & 10.38 & 10.2 & 10.3 & Yes & $0^{\dagger}$ & $0.11^{\dagger}$ & & & $10.27^{\dagger}$ & $10.38^{\dagger}$ & & \\
\hline
\end{tabular}

* = fall-in, $\dagger=$ flow-in, basal and midcore.

Table T2. Summary of coring disturbances, Expedition 340.

\begin{tabular}{|c|c|c|c|c|c|c|c|c|c|}
\hline Hole & $\begin{array}{l}\text { Core sections } \\
\text { in hole }\end{array}$ & $\begin{array}{l}\text { Disturbed } \\
\text { core sections }\end{array}$ & $\begin{array}{c}\text { APC } \\
\text { recovered }(m)\end{array}$ & $\begin{array}{c}\text { Disturbed } \\
\text { (fall-in) (m) }\end{array}$ & $\begin{array}{l}\text { Possibly disturbed } \\
\text { (fall-in) (m) }\end{array}$ & $\begin{array}{c}\text { Disturbed } \\
\text { (flow-in) (m) }\end{array}$ & $\begin{array}{c}\text { Possibly } \\
\text { disturbed } \\
\text { (flow-in) }(\mathrm{m})\end{array}$ & $\begin{array}{c}\text { Disturbed } \\
\text { minimum (\%) }\end{array}$ & $\begin{array}{c}\text { Disturbed } \\
\text { maximum (\%) }\end{array}$ \\
\hline \multicolumn{10}{|l|}{$340-$} \\
\hline U1393A & 6 & 6 & 4.37 & 0.01 & 0 & 1.36 & 3 & 31.4 & 100.0 \\
\hline U1394A & 22 & 11 & 24.17 & 0 & 0 & 10 & 0 & 41.4 & 41.4 \\
\hline U1394B & 117 & 46 & 137.42 & 1.51 & 0.12 & 38.25 & 0 & 28.9 & 29.0 \\
\hline U1395A & 102 & 18 & 124.29 & 0.3 & 0.09 & 8.12 & 7.89 & 6.8 & 13.2 \\
\hline U1395B & 106 & 26 & 127.51 & 0 & 2.98 & 16.18 & 1.68 & 12.7 & 16.3 \\
\hline U1396A & 114 & 7 & 140.51 & 0 & 0.14 & 0.92 & 0 & 0.7 & 0.8 \\
\hline U1396B & 8 & 1 & 10 & 0.02 & 0.06 & 4.34 & 0 & 43.6 & 44.2 \\
\hline U1396C & 119 & 8 & 145.92 & 0.02 & 0.06 & 4.34 & 0 & 3.0 & 3.0 \\
\hline U1397A & 110 & 55 & 118.1 & 1.81 & 3.73 & 22.88 & 7.51 & 20.9 & 30.4 \\
\hline U1397B & 96 & 47 & 108.07 & 3.36 & 0 & 34.85 & 0 & 35.4 & 35.4 \\
\hline U1398A & 74 & 29 & 86.98 & 1.89 & 7.63 & 12.92 & 0.59 & 17.0 & 26.5 \\
\hline U1398B & 156 & 103 & 172.11 & 3.49 & 4.73 & 81.63 & 4.57 & 49.5 & 54.9 \\
\hline U1399A & 185 & 86 & 210.45 & 1.99 & 2.2 & 56.37 & 5.77 & 27.7 & 31.5 \\
\hline U1399B & 162 & 70 & 184.16 & 2.2 & 1.47 & 41.7 & 8.84 & 23.8 & 29.4 \\
\hline U1400A & 56 & 44 & 51.8 & 0.9 & 1 & 31.39 & 1.28 & 62.3 & 66.7 \\
\hline U1400B & 187 & 45 & 215.19 & 0.62 & 2.17 & 30.71 & 2.29 & 14.6 & 16.6 \\
\hline U1400C & 150 & 27 & 180.35 & 6 & 2.35 & 7.16 & 8.5 & 7.3 & 13.3 \\
\hline U1401A & 16 & 11 & 14.45 & 0 & 0.2 & 1.87 & 2.92 & 12.9 & 34.5 \\
\hline U1401B & 13 & 8 & 12.42 & 0.13 & 2 & 1.21 & 0.64 & 10.8 & 32.0 \\
\hline U1401C & 12 & 9 & 10.44 & 2.41 & 0 & 0 & 2.34 & 23.1 & 45.5 \\
\hline U1401D & 7 & 0 & 9.12 & 0 & 0 & 0 & 0 & 0.0 & 0.0 \\
\hline $\begin{array}{r}\text { Total (\%): } \\
\text { Average: }\end{array}$ & 1818 & $657(36.1)$ & 2087.83 & $26.66(1.3)$ & $30.93(1.5)$ & $406.2(19.5)$ & $57.82(2.8)$ & 22.6 & 31.7 \\
\hline
\end{tabular}

